\title{
The low-end of the black hole mass function at cosmic dawn
}

\author{
Alessandro Trinca ${ }^{1,2,3 \star}$, Raffaella Schneider ${ }^{1,2,3,5}$, Rosa Valiante ${ }^{2,3}$, Luca Graziani ${ }^{1,3,4}$, \\ Luca Zappacosta ${ }^{2}$, Francesco Shankar ${ }^{6}$ \\ ${ }^{1}$ Dipartimento di Fisica, “Sapienza” Università di Roma, Piazzale Aldo Moro 2, 00185 Roma, Italy \\ ${ }^{2}$ INAF/Osservatorio Astronomico di Roma, Via di Frascati 33, 00040 Monte Porzio Catone, Italy \\ ${ }^{3}$ INFN, Sezione Roma1, Dipartimento di Fisica, “Sapienza” Università di Roma, Piazzale Aldo Moro 2, 00185, Roma, Italy \\ ${ }^{4}$ INAF/Osservatorio Astrofisico di Arcetri, Largo E. Fermi 5, 50125 Firenze, Italy \\ ${ }^{5}$ Sapienza School for Advanced Studies, Viale Regina Elena 291, 00161 Roma, Italy \\ ${ }^{6}$ Department of Physics and Astronomy, University of Southampton, Highfield, SO17 1BJ, UK
}

Accepted 2022 January 5. Received 2022 January 5; in original form 2021 July 31

\begin{abstract}
Understanding the formation and growth of supermassive black holes (SMBHs) at high redshift represents a major challenge for theoretical models. In this work we investigate the early evolution of the first SMBHs by constraining their distribution in mass and luminosity at $z>4$. In particular, we focus on the poorly explored low-mass end of the nuclear black hole $(\mathrm{BH})$ distribution down to $z \simeq 4$, and explore its connection with the nature of the first $\mathrm{BH}$ seeds and the processes governing their mass growth. To this aim, we have developed CAT (Cosmic Archaeology Tool), a new semi-analytic model that describes the formation of the first stars and black holes in a self-consistent way and follows the co-evolution of nuclear $\mathrm{BHs}$ and their host galaxies for a representative population at $z>4$. We find that current observational constraints favour models where the growth of $\mathrm{BH}$ seeds is Eddington limited and occurs at the Bondi-Hoyle-Lyttleton rate or where super-Eddington accretion occurs via a slim disk during gas rich galaxy mergers. The main difference between these two model variants lies at the low-end of the predicted mass and luminosity functions at $4 \leq z \leq 6$, where a clear gap appears in the first model, reflecting the stunted growth of light $\mathrm{BH}$ seeds formed as remnants of the first stars. Detecting this signature will be extremely challenging even for the future generation of space observatories, such as JWST, Athena and Lynx.
\end{abstract}

Key words: galaxies: active - galaxies: formation - galaxies: evolution - galaxies: highredshift - quasars: supermassive black holes - black hole physics

\section{INTRODUCTION}

In the last decade hyper-luminous quasars $\left(L \geq 10^{47} \mathrm{erg} / \mathrm{s}\right)$ have been observed up to $z>7$, suggesting that Super Massive BHs (SMBHs) with masses as high as ten billion solar have already formed when the Universe was less than 1 Gyr old. The most massive detected system, SDSS J0100+2802, is a quasar observed at $z \sim 6.3$ (Wu et al. 2015) with an estimated mass of $\mathrm{M}_{\mathrm{BH}}=(1.2 \pm 0.19) \times$ $10^{10} \mathrm{M}_{\odot}$, while the most distant quasars are ULAS $\mathrm{J} 1342+0928$ ( $\mathrm{z}=7.54$, Bañados et al. 2018) powered by a central BH with $\mathrm{M}_{\mathrm{BH}} \sim 8 \times 10^{8} \mathrm{M}_{\odot}$, Pōniuā'ena $(\mathrm{J} 1007+2115)$ at $z=7.52\left(\mathrm{M}_{\mathrm{BH}}=\right.$ $1.5 \times 10^{9} M_{\odot}$, Yang et al. 2020) and the recently reported J03131806 at $z=7.64\left(\mathrm{M}_{\mathrm{BH}}=1.6 \times 10^{9} M_{\odot}\right.$, Wang et al. 2021).

Explaining how these extreme objects form and grow during the first billion years of cosmic history still represents a major

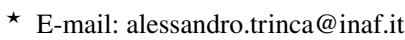

challenge for theoretical models (for thorough reviews see Volonteri 2010; Valiante et al. 2017; Inayoshi et al. 2020). Therefore, it is crucial to understand what is the nature of SMBH first progenitors (generally referred to as BH "seeds") and how these efficiently grow through gas accretion and mergers. Many possible scenarios for the formation of $\mathrm{BH}$ seeds have been proposed in the literature so far.

Light seeds, with a $\mathrm{BH}$ mass $\sim 100 \mathrm{M}_{\odot}$, are supposed to be the remnants of the first generation of metal-free (Population III/Pop III) stars and are expected to form with masses ranging between a few 10 s to a few $100 \mathrm{~s}_{\odot}$, inside dark matter (DM) minihalos $\left(M_{\text {halo }} \sim 10^{5}-10^{6} \mathrm{M}_{\odot}\right)$ at very high redshifts $(z \geq 20$, see Bromm 2013 for a review, and Hirano et al. 2014; Hirano et al. 2015; Hosokawa et al. 2016; Sugimura et al. 2020 for more recent works).

Collisions between stars inside dense star clusters are instead supposed to give birth to intermediate mass or medium-weight $\mathrm{BH}$ seeds, with a mass $\sim 1000 \mathrm{M}_{\odot}$ (Omukai et al. 2008; Devecchi \& Volonteri 2009; Katz et al. 2015; Sakurai et al. 2017; Reinoso et al. 
2018; Reinoso et al. 2019; Sassano et al. 2021), although even more massive BH seeds could originate from merger episodes between stellar mass BHs (aided by strong gas inflows) inside similarly dense environments (Lupi et al. 2014; Boco et al. 2020).

Recent studies have also suggested that very massive BHs $\left(\sim 10^{5}-10^{6} \mathrm{M}_{\odot}\right)$, usually referred to as heavy seeds, can form via the direct collapse of a supermassive star. The above mechanism is supposed to take place inside dark matter halos with virial temperatures $T_{\text {vir }}>10^{4} \mathrm{~K}$, where gas cooling and fragmentation is suppressed by their metal-poor composition and $\mathrm{H}_{2}$ photo-dissociation caused by Lyman-Werner (LW) radiation (Omukai 2001; Bromm \& Loeb 2003; Wise et al. 2008; Regan \& Haehnelt 2009; Hosokawa et al. 2012; Latif et al. 2013; Inayoshi et al. 2014; Latif et al. 2016; Chon et al. 2016; Becerra et al. 2018) or by dynamical heating (Wise et al. 2019; Lupi et al. 2021) associated with strong gas inflows (Lodato \& Natarajan 2006, 2007; Mayer et al. 2010, 2015; Mayer \& Bonoli 2019; Haemmerlé et al. 2019).

Although the formation rate of heavy $\mathrm{BH}$ seeds is yet to be established (see Valiante et al. 2017; Inayoshi et al. 2020 and references therein) and their growth efficiency might depend on the formation site inside the host galaxy (e.g. Chon et al. 2021), they represent one of the most promising formation scenarios to explain the existence of SMBHs at $z \geq 6$ without invoking super-Eddington accretion rates. It has been shown, in fact, that starting from a seed of $\mathrm{M}_{\mathrm{BH}} \sim 10^{5} \mathrm{M}_{\odot}$, a BH can reach the observed mass of high-redshift SMBHs through classical Eddington-limited growth (Valiante et al. 2017; Sassano et al. 2021). Many studies also pointed out that less massive seeds would require persistent accretion of gas at the Eddington rate during all their existence to reach a billion solar masses in less than 1 Gyr (Volonteri 2010; Madau et al. 2014; Bañados et al. 2018). Alternatively, intermittent gas accretion at super- or hyper-Eddington rates may efficiently grow lower mass seeds (Pezzulli et al. 2016; Inayoshi et al. 2016; Pezzulli et al. 2017b; Pacucci et al. 2017; Takeo et al. 2018), provided that suitable conditions are met in their circum-nuclear regions at high- $z$ (see the discussion in Mayer 2018 and references therein).

Despite the significant progresses made in their theoretical description, discriminating the nature of $\mathrm{BH}$ seeds by electromagnetic observations may be very challenging, for a number of reasons: $\mathrm{BH}$ seeds keep memory of their birth conditions and genetic origin as long as they live in isolation, accreting gas from their host galaxy. For seed progenitors of $z \sim 6-7 \mathrm{SMBHs}$, these conditions are only met at $z>10$ for $<100-150$ Myr since their formation (Valiante et al. 2018a). In addition, light BH seeds may be too faint to be detectable, even with upcoming facilities, at least in Eddington-limited growth scenarios (Valiante et al. 2018b). Hence, the only chance to constrain their nature would be to detect the gravitational waves emitted during their binary coalescence through third generation ground-based detectors, such as the Einstein Telescope ${ }^{1}$ (Valiante et al. 2020). Detecting the electromagnetic emission from rapidly growing heavy $\mathrm{BH}$ seeds appears more promising, and photometric selection techniques have been envisaged to help identify the more promising candidates (Pacucci et al. 2015, 2016; Natarajan et al. 2017; Valiante et al. 2018b).

An alternative way to constrain the early evolution of black holes is by shedding light onto the low-mass end of the black hole mass function at high- $z$ (Volonteri et al. 2008; Volonteri \& Natarajan 2009; Ricarte \& Natarajan 2018b; Piana et al. 2021), which is

\footnotetext{
1 http://www.et-gw.eu/
}

supposed to be very sensitive to the nature of $\mathrm{BH}$ seeds and their growth mode.

Several optical/Near-Infrared surveys such as the Sloan Digital Sky Survey $\left(\mathrm{SDSS}^{2}\right)$, the UKIRT Infrared Deep Sky Survey $\left(\mathrm{UKIDSS}^{3}\right)$, the DECam Legacy Survey $\left(\mathrm{DECaLS}^{4}\right)$ and the Panoramic Survey Telescope and Rapid Response System (PanSTARRS $^{5}$ ) gave a fundamental contribution to the detection of luminous quasars at redshift $z \geq 6$, characterizing the bright end of their luminosity function (LF, Willott et al. 2010a; Bañados et al. 2016). At very high redshift, however, these objects are rare and presumably trace extremely biased regions of the Universe.

Large-scale cosmological simulations predict that a much larger population of fainter AGNs, powered by less massive BHs, is assembling and growing together with their host galaxies at these cosmic epochs (Di Matteo et al. 2012; Vogelsberger et al. 2014; Schaye et al. 2015; Feng et al. 2016). This population has eluded direct detection until recent surveys, in particular the Subaru High-z Exploration of Low-Luminosity Quasars (SHELLQS), have started to explore fainter magnitudes, unveiling a large sample of lowluminosity quasars. These findings have raised the total number of observed AGNs at $z>6$ above 200, constraining for the first time the faint-end of their LF (Matsuoka et al. 2018; Matsuoka et al. 2019).

In recent years, a number of semi-analytical models have been applied to investigate observational signatures of $\mathrm{BH}$ seeds and of their early co-evolution with their host galaxies (Volonteri et al. 2008; Somerville et al. 2008; Devecchi et al. 2012; Salvaterra et al. 2012; Yue et al. 2013; Bonoli et al. 2014; Valiante et al. 2016; Valiante et al. 2018b; Pezzulli et al. 2016, 2017a; Ricarte \& Natarajan 2018a,b; Piana et al. 2021; Dayal et al. 2020). In these models the evolution of the baryonic components of dark matter halos is described through physically- and/or observationally-motivated prescriptions and the growth of dark matter halos can be either generated analytically or extracted from numerical simulations (Baugh 2006; De Lucia 2019).

In this work we investigate the mass function (MF) of supermassive black holes, and the corresponding AGNs LF, by following their redshift evolution from the epoch of $\mathrm{BH}$ seeds formation down to $z=4$. In particular, we focus on the large population of lowmass faint systems that will be targeted by upcoming facilities, such as the James Webb Space Telescope (JWST), the Advanced Telescope for High Energy Astrophysics (ATHENA) and the Lynx Xray Observatory. We also investigate whether initial $\mathrm{BH}$ seed masses and their growth rates leave an imprint on the low-mass (faint) end of the MF (LF) that may potentially be used to discriminate among different scenarios. To this aim, we developed CAT (Cosmic Archaeology Tool), a semi-analytical model that describes structure formation in the first billion years of cosmic evolution following the hierarchical growth of dark matter halos, their stellar and gas content, and their nuclear black holes. CAT can account for a wide range of halo masses: from the $10^{6} \mathrm{M}_{\odot}$ mini-halos hosting the collapse of the first stars at $z=20-30$, up to the largest galaxies with $M_{h} \sim 10^{12}-10^{14} \mathrm{M}_{\odot}$ where the most powerful quasars at $z=4-7$ are supposed to reside. CAT can explore a statistics which is still prohibitively expensive for cosmological hydro-dynamical simulations (Springel et al. 2005; Di Matteo et al. 2005, 2017;

\footnotetext{
2 https://www.sdss.org/

3 https://www.nottingham.ac.uk/astronomy/UDS/

4 https://www.legacysurvey.org/decamls/

5 https://panstarrs.stsci.edu/
} 
Sijacki et al. 2007, 2015; DeGraf et al. 2012, 2015; Hirschmann et al. 2014; McAlpine et al. 2017; Weinberger et al. 2017; Habouzit et al. 2019), and can be achieved only through zoom-in techniques on a small number of systems pre-selected in very large lowerresolution simulations (Regan et al. 2019; Lupi et al. 2019; Zhu et al. 2020).

CAT builds on our semi-analytical model GAMETEQSODUST (GQD), that was successfully applied to study the co-evolution of SMBHs and their host galaxies at $z \geq 6$ (Valiante et al. 2011, 2014, 2016). In the present version, СAT enables to follow the formation of both light and heavy BH seeds depending on the environmental properties and to explore their contribution to the BH MF (AGNs LF) across a wide range of redshifts, mass (luminosity) scales and physical parameters, quantifying the conditions for seed formation and their mass growth rate. This kind of investigation is certainly beyond the modelling capabilities of current large-scale cosmological hydrodynamic simulations, which usually adopt simplified prescriptions for $\mathrm{BH}$ seeding and assume a fixed $\mathrm{BH}$ seed mass that is planted in dark matter halos above a given threshold mass, independently of their internal properties (Di Matteo et al. 2012; Vogelsberger et al. 2014; Schaye et al. 2015; Khandai et al. 2015; Feng et al. 2016). More physically sound $\mathrm{BH}$ seeding prescriptions have been adopted in smaller-scale or zoom-in simulations (Bellovary et al. 2011; Habouzit et al. 2017; Tremmel et al. 2017; Huang et al. 2020), but at the price of being unable to simultaneously explore the low- and high-mass (luminosity) ends of the BH MF (LF).

To explore how different high- $z$ formation scenarios leave their imprints on the low-mass (luminosity) end of the BH MF (LF) at $z \geq 4$, we have run a large set of simulations on a grid of halo merger histories extending over several orders of magnitude in mass at $z=4$ using the galaxy formation model GALFORM (Cole et al. 2000; Parkinson et al. 2008). Our model predictions are then compared with the observed properties of galaxies and AGNs in view of future observations which could shed some light in discriminating among different BH seeding and growth scenarios.

The paper is organized as follows. In Section 2 we introduce and describe the model while in Section 3 we show how we calibrate the model to set the free parameters that govern star formation and black hole growth. The results are presented in Section 4, where we also compare our findings with the most recent observations and theoretical models. Finally, in Section 5 we discuss and summarize our main results.

\section{THE COSMIC ARCHAEOLOGY TOOL}

In this section we illustrate the Cosmic Archaeology Tool (CAT) adopted in the present work. First, we present the galaxy formation model GALFORM and how its dark matter halo merger tree algorithm was properly adapted to generate the sample of dark matter halos. Second, we describe the main features of GQD that has been imported in CAT in order to follow the evolution of halo baryonic components (gas, stars, and nuclear black holes), along with the major improvements we introduced in the present work.

In what follows we assume a Lambda cold dark matter $(\Lambda \mathrm{CDM})$ cosmological model with the following parameters: $\Omega_{\Lambda}=$ $0.685, \Omega_{\mathrm{m}}=0.315, h=0.674, \Omega_{\mathrm{b}}=0.05$ (Planck Collaboration et al. 2018) so that the age of the Universe at the final redshift $z=4$ is $t_{\mathrm{H}} \sim 1.53 \mathrm{Gyr}$.

\subsection{Halo Merger Trees}

GALFORM is a semi-analytic model of galaxy formation that reconstructs the hierarchical merger history of a given dark matter halo, also referred to as halo merger tree. We adopted the improved Monte Carlo algorithm developed by Parkinson et al. (2008), based on the Extended Press Schechter theory (EPS) and properly tuned to obtain an accurate agreement with N-body simulations, in particular with the results of the Millennium Simulation (MS, Springel et al. 2005). Here we briefly summarize the merger trees reconstruction algorithm.

Starting from a target halo with a given mass at redshift $z_{0}$, GALFORM follows its evolution backward in time reconstructing its progenitors. The key point of this process is the conditional mass function given by the EPS theory (Cole et al. 2000):

$$
\begin{gathered}
f\left(M_{1} \mid M_{2}\right) d \ln M_{1}=\sqrt{\frac{2}{\pi}} \frac{\sigma_{1}^{2}\left(\delta_{1}-\delta_{2}\right)}{\left[\sigma_{1}^{2}-\sigma_{2}^{2}\right]^{3 / 2}} \times \\
\exp \left[-\frac{1}{2} \frac{\left(\delta_{1}-\delta_{2}\right)^{2}}{\left(\sigma_{1}^{2}-\sigma_{2}^{2}\right)}\right]\left|\frac{d \ln \sigma}{d \ln M_{1}}\right| d \ln M_{1},
\end{gathered}
$$

where $f\left(M_{1} \mid M_{2}\right)$ represents the fraction of mass of halos of mass $M_{2}$ at redshift $z_{2}$ that is contained in progenitor halos of mass $M_{1}$ at an earlier redshift $z_{1}$. The values $\delta_{1}$ and $\delta_{2}$ are instead the linear density thresholds for collapse at these two redshifts and $\sigma(M)$ represents the rms linear density fluctuation in spheres containing a mass $M$, extrapolated to $z=0$, with $\sigma_{1 / 2} \equiv \sigma\left(M_{1 / 2}\right)$. Starting from Eq. (1) it is possible to obtain the mean number of halos of mass $M_{1}$ into which a halo of mass $M_{2}$ splits after a step up in redshift $d z_{1}$, that is

$\frac{d N}{d M_{1}}=\frac{1}{M_{1}} \frac{d f}{d z_{1}} \frac{M_{2}}{M_{1}} d z_{1} \quad\left(M_{1}<M_{2}\right)$.

Hence, the halo is decomposed into its progenitors and the process is repeated on each new halo at previous redshift steps up to a final value $z_{\max }$, building up a complete tree. Although the above algorithm produces merger trees with statistical properties in good agreement with those obtained through detailed N-body simulations, it should be noted that the classical EPS theory systematically underestimates the mass of the most massive progenitor halos with increasing redshift (Cole et al. 2008). For this reason a perturbing function $G\left(\sigma_{1} / \sigma_{2}, \delta_{2} / \sigma_{2}\right)$ was introduced, leading to a modification of Eq. (2) as follows:

$\frac{d N}{d M_{1}} \rightarrow \frac{d N}{d M_{1}} G\left(\sigma_{1} / \sigma_{2}, \delta_{2} / \sigma_{2}\right)$,

where

$G\left(\sigma_{1} / \sigma_{2}, \delta_{2} / \sigma_{2}\right)=G_{o}\left(\frac{\sigma_{1}}{\sigma_{2}}\right)^{\gamma_{1}}\left(\frac{\delta_{2}}{\sigma_{2}}\right)^{\gamma_{2}}$

$G_{0}, \gamma_{1}$ and $\gamma_{2}$ are free parameter calibrated to reproduce the MS conditional mass function.

We used the GALFORM algorithm to simulate the formation histories of DM halos with masses in the range $\left[10^{9}-10^{14}\right] \mathrm{M}_{\odot}$ at $z_{\min }=4$. This allows us to explore both the low-mass end of halo population and halos as large as $M_{\text {halo }} \sim 10^{13} \mathrm{M}_{\odot}$ at $z \geq 6$, that are assumed to host the first SMBHs that power the observed highly luminous quasars (Fan et al. 2003; Valiante et al. 2011, 2016). We divide this mass interval into 11 logarithmically spaced bins with size 0.5 . For each bin, we consider a final halo of mass equal to the central bin value and we use it as a starting point for the GALFORM code to simulate 10 independent halo merger trees. 
Once the total merger tree sample has been generated, the resulting redshift dependent mass distributions of each mass bin are weighted according to the number density of DM halos at redshift $z=4$, as given by the Sheth and Tormen mass function (Sheth et al. 2001). In this way, the normalized sample is representative of the halo population at redshifts $z \geq 4$. It is important to stress that the limited number of merger tree realizations simulated for each final halo mass might, in principle, limit the allowed variability in the evolution of the corresponding galaxy properties. This is especially true for the lowest final halo masses. By doubling the number of merger tree simulations we find that the redshift evolution of mean galaxy properties does not change, showing that the current sampling scheme provides enough statistical variance even for the lowest halo mass bins.

\subsubsection{The Dark Matter Mass Resolution}

In the $\Lambda \mathrm{CDM}$ cosmological model, where larger structures form hierarchically through successive mergers of smaller ones, the first stars are expected to form inside the so-called minihalos, i.e. small dark matter halos with $M_{\text {halo }} \sim 10^{5}-10^{6} \mathrm{M}_{\odot}$, at redshift $z \sim 20-30$. Here we classify as minihalos systems with virial temperature in the range $1200 \mathrm{~K} \leq T_{\text {vir }} \leq 10^{4} \mathrm{~K}$, where $T_{\text {vir }}$ can be expressed as a function of redshift $z$ as (Bromm 2013):

$T_{\mathrm{vir}} \simeq 2 \times 10^{3} \mathrm{~K}\left(\frac{\mathrm{M}_{\text {halo }}}{10^{6} \mathrm{M}_{\odot}}\right)^{2 / 3}\left(\frac{1+\mathrm{z}}{20}\right)$.

In these objects, where the gas temperature is below the threshold of $10^{4} \mathrm{~K}$ for efficient cooling due to atomic hydrogen, the pristine gas can still cool down and fragment through the roto-vibrational emission of molecular hydrogen $\left(\mathrm{H}_{2}\right)$, giving birth to the first generation of Pop III stars. Due to their birth conditions, Pop III stars are expected to be massive and a large fraction of these will terminate their life as a stellar-mass $\mathrm{BH}$, providing the first light $\mathrm{BH}$ seeds (Valiante et al. 2016). Hence, any model attempting to describe the build-up of SMBHs from growing BH seeds must be able to describe the formation of the first stars and BHs in high- $z$ minihalos. For this reason, while running GALFORM, we set a minimum DM halo mass of $M_{\text {res }}=M_{\text {halo }}\left(T_{\text {vir }}=1200 \mathrm{~K}\right)$. We assumed $1200 \mathrm{~K}$ as the minimum virial temperature for the onset of efficient $\mathrm{H}_{2}$ cooling following the work of Haiman et al. (1996). ${ }^{6}$

In Fig. 1 the mass resolution as a function of redshift adopted in CAT (solid orange line) is compared to the one adopted in GQD (red dashed line) for a final halo mass of $10^{13} \mathrm{M}_{\odot}$ at $z=6.4$. The solid brown line shows the redshift dependent minimum mass of atomiccooling halos, $M_{\text {halo }}\left(T_{\text {vir }}=10^{4} \mathrm{~K}\right)$, so that the yellow shaded region represents the range of masses of the minihalos population. It is clear that the increased resolution of CAT largely improves the statistics of minihalos with respect to GQD and allows us to follow their evolution all the way down to $z=4$. We obtain maximal improvements especially in the description of the most massive halos since in CAT the mass resolution at a given redshift is fixed, while in GQD it depends on the final halo mass.

6 Note, however, that in our model not all mini-halos are equally efficient at forming stars as the fraction of available cold gas depends on the strength of the illuminating UV background, the redshift and the metallicity (see eq. $6)$.

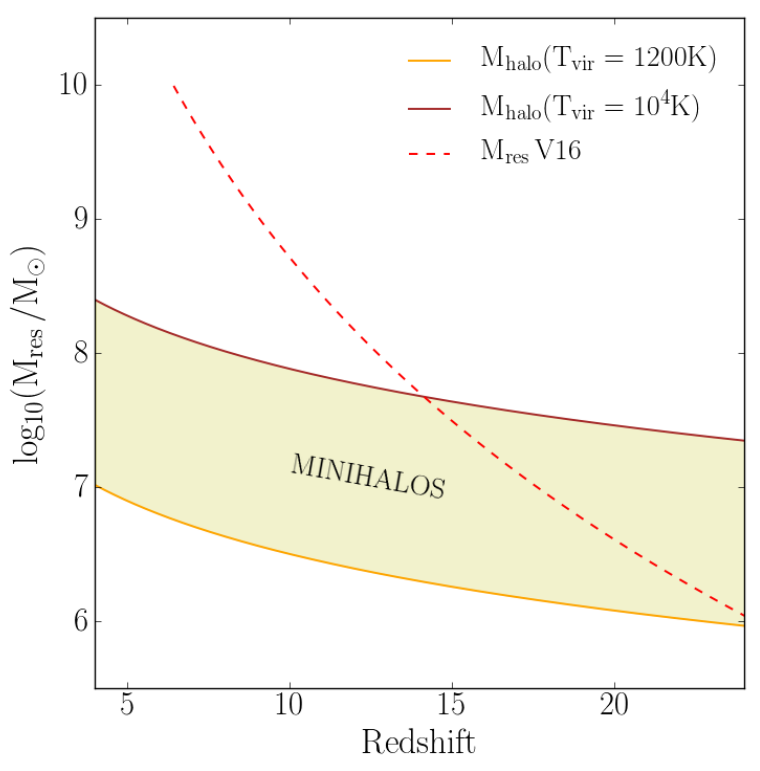

Figure 1. The mass resolution adopted when generating GALFORM merger trees (orange solid line) is compared to the merger tree mass resolution of Valiante et al. (2016, red dashed line), where a final halo mass of $10^{13} \mathrm{M}_{\odot}$ at $z=6.4$ is assumed. For comparison, we also show the redshift dependent minimum mass of atomic cooling halos (brown solid line) so that the yellow shaded region illustrates the masses of dark matter minihalos with $1200 \mathrm{~K} \leq$ $T_{\text {vir }} \leq 10^{4} \mathrm{~K}$.

\subsubsection{Multiple Mergers}

In its original setup, GALFORM merger trees are generated according to an adaptive number of steps that ensures their binarity (Cole et al. 2000). However, to follow the baryonic evolution, we re-grid all the merger trees, according to Valiante et al. (2016), on $N_{\mathrm{t}}=800$ time-steps logarithmically spaced in expansion factor between $z=24$ and $z=4$, so that the time interval between two simulation snapshots is $\sim 0.5 \mathrm{Myr}$ at $z \sim 20$, and $\sim 4 \mathrm{Myr}$ at $z \sim 4$. A consequence of this process is that the binary structure of the merger trees is no longer ensured since a given dark matter halo at a redshift step $d z_{i}$ can be formed as a result of the merger of more than two halos that were in place at a higher redshift $d z_{i+1}$, enabling the occurrence of what we call multiple mergers.

It is common to define as major merger between two DM halos a merger event where the mass ratio $\mu$ between the least and most massive halo is higher than a chosen threshold value. Because of the loss of binarity, here we classify as major mergers interactions where the mass ratio between the first and the second most massive halos among the merging ones is $\mu>1 / 10$. This choice appears to be conservative, possibly leading to an underestimation of the number of major mergers, due to the lack of information about the order of mergers in multiple interactions occurring in a single timestep.

\subsection{Baryonic evolution}

In CAT, the evolution of the baryonic component is governed by the same physical prescriptions adopted in GQD, that we briefly summarize below. We will focus on $\mathrm{BH}$ evolution and on the new 
features introduced in this work, referring the reader to Valiante et al. $(2014,2016)$ for a more detailed description.

GQD was developed to investigate the formation history of the most extreme quasars observed at $z \geq 6$ and their host galaxies. To this aim, it follows the evolution of gas, stars, metals, and dust in each progenitor galaxy along a merger tree, tracking the process of star formation and the enrichment of the interstellar medium (ISM) due to Asymptotic Giant Branch (AGB) stars and Supernovae (SNe) by means of mass- and metallicity-dependent yields. The model follows a two-phase ISM environment where dust grains can both be destroyed by SN shocks expanding in the diffuse hot medium and can grow in mass by accreting gas-phase metals in warm dense gas (see Valiante et al. 2014; de Bennassuti et al. 2014, for details) ${ }^{7}$ Mechanical feedback due to SN explosions and energy deposition associated with $\mathrm{BH}$ growth is also considered. The energy released by these processes couples with the gas, eventually removing a significant fraction of the galactic reservoir though energy-driven galactic scale winds (Valiante et al. 2012).

So far, GQD has been applied to study the formation of single $z>6$ quasars, hosting $>10^{9} M_{\odot}$ SMBHs, in association with the evolution of their host galaxies (Valiante et al. 2011, 2014), BH seeds origin/properties (Valiante et al. 2016; Valiante et al. 2018a,b; Sassano et al. 2021), earliest binary BHs formation (Valiante et al. 2020) and different gas accretion regimes (Pezzulli et al. 2016, 2017a; Pezzulli et al. 2017b). In the present work we are instead interested in studying a population of galaxies and their nuclear BHs across a broad range of masses and populating a less biased region of the Universe down to $z=4$.

\subsubsection{Star formation and feedback}

In the same spirit of GQD, CAT follows the evolution of the gas mass inside each galaxy during the whole DM halo assembly. Following halo virialization, the gas is accreted onto the newly collapsed halo and cools down to efficiently trigger star formation. The resulting fraction of gas mass is set by the balance between gas cooling and dynamical times. Inside each galaxy the star formation rate (SFR) is computed as:

$\mathrm{SFR}=f_{\text {cool }} M_{\mathrm{gas}} \epsilon_{\mathrm{SF}} / \tau_{\mathrm{dyn}}$,

where $M_{\mathrm{gas}}$ is the available gas mass reservoir, $\epsilon_{\mathrm{SF}}$ is the star formation efficiency per unit of time and $\tau_{\text {dyn }}=\left[R_{\text {vir }}^{3} /\left(G M_{\text {halo }}\right)\right]^{1 / 2}$ is the dynamical time of the system. The SF efficiency $\epsilon_{\mathrm{SF}}$ represents a free parameter of the model and is calibrated as discussed in Section 3. $f_{\text {cool }}$ quantifies the reduced cooling efficiency in minihalos, where it depends on the halo virial temperature, redshift, gas metallicity and intensity of the illuminating LW radiation, as previously implemented by Valiante et al. (2016); de Bennassuti et al. (2017). Conversely, in atomic cooling halos we set $f_{\text {cool }}=1$. As described in Valiante et al. (2016), we compute at each redshift $z_{\text {obs }}$ the global LW cumulative background flux at the observed frequency $v_{\mathrm{obs}}$ as

$J\left(v_{\mathrm{obs}}, z_{\mathrm{obs}}\right)=\frac{\left(1+z_{\mathrm{obs}}\right)^{3}}{4 \pi} \int_{z_{\mathrm{obs}}}^{z_{\mathrm{max}}} d z c\left|\frac{d t}{d z}\right| \epsilon\left(v_{z}, z\right) e^{-\tau_{\mathrm{H}_{2}}\left(v_{\mathrm{obs}}, z_{\mathrm{obs}}, z\right)}$

\footnotetext{
7 The same physical prescriptions for metal and dust enrichment have been adopted in semi-numerical models (Mancini et al. 2015, 2016) as well as in cosmological hydrodynamical simulations (Graziani et al. 2020) and provide good agreement with the observed dusty galaxy population at $z \geq 4$.
}

where $\epsilon\left(v_{z}, z\right)$ is the comoving emissivity in the LW band at redshift $z$, which is obtained summing over all the emitting sources, both stars and accreting BHs. In Eq. 7, $\tau_{\mathrm{H}_{2}}$ is the $\mathrm{H}_{2}$ optical depth in the LW band (for a detailed description of its calculation see Valiante et al. 2016), while $z_{\max }$ is the maximum redshift from which a LW photon emitted by a source at $z>z_{\text {obs }}$ can reach the observer before being redshifted outside the LW band.

Finally, we account for the effects of photo-heating feedback by suppressing star formation in haloes with virial temperatures below the temperature of the intergalactic medium (IGM), $T_{\mathrm{vir}}<T_{\mathrm{IGM}}$. We consider $T_{\mathrm{IGM}}=Q_{\mathrm{HII}} T_{\text {reio }}+\left(1-Q_{\mathrm{HII}}\right) T_{\mathrm{HI}}$, where $T_{\text {reio }}=2 \times 10^{4} \mathrm{~K}$, $T_{\mathrm{HI}}=0.017(1+z)^{2}$ and the filling factor of HII regions, $Q_{\mathrm{HII}}$, is computed as in (Valiante et al. 2016).

The abundance of gas inside each galaxy is also affected by mechanical feedback associated with $\mathrm{SN}$ explosions and $\mathrm{BH}$ accretion, whose released energy drives massive outflows of gas out of the galaxy. The total gas ejection rate, $\dot{M}_{\mathrm{ej}}$ is described as:

$\dot{M}_{\mathrm{ej}}=\dot{M}_{\mathrm{ej}, \mathrm{SN}}+\dot{M}_{\mathrm{ej}, \mathrm{AGN}}$

where $\dot{M}_{\mathrm{ej}, \mathrm{SN}}$ and $\dot{M}_{\mathrm{ej}, \mathrm{AGN}}$ are the SN- and AGN-driven outflow rates, respectively, defined as:

$\dot{M}_{\mathrm{ej}, \mathrm{SN}}=\frac{2 E_{\mathrm{SN}} \epsilon_{\mathrm{w}, \mathrm{SN}} R_{\mathrm{SN}}(t)}{v_{\mathrm{e}}^{2}}$,

and

$\dot{M}_{\mathrm{ej}, \mathrm{AGN}}=2 \epsilon_{\mathrm{w}, \mathrm{AGN}} \epsilon_{r} \dot{M}_{\mathrm{accr}}\left(\frac{c}{v_{\mathrm{e}}}\right)^{2}$.

In Eq. $9 R_{\mathrm{SN}}(t)$ is the $\mathrm{SN}$ explosion rate, which depends on the SF history and on the nature of the stellar populations hosted by each galaxy. For Pop III stars, we stochastically sample the Initial Mass Function (IMF) in each SF episode and $R_{\mathrm{SN}}(t)$ depends on the number of stars formed at each time in each galaxy. For Pop II stars, instead, we assume a fully sampled IMF and $R_{\mathrm{SN}}(t)=$ $1.25 \times 10^{-2} M_{\odot}^{-1}$ (see Section 2.3.1 for more details). In Eq. $9 E_{\mathrm{SN}}$ represents the explosion energy per $\mathrm{SN}$, assumed to be $2.7 \times 10^{52}$ erg for Pop III stars and $1.2 \times 10^{51}$ erg for Pop II stars.

The terms $\dot{M}_{\text {accr }}$ and $\epsilon_{r}$, in Eq. 10, are instead the gas accretion rate and the AGN radiative efficiency, described in Section 2.3.4. In both expressions, $v_{\mathrm{e}}=\left(2 G M / R_{\mathrm{vir}}\right)^{1 / 2}$ is the escape velocity of the galaxy while $\epsilon_{\mathrm{w}, \mathrm{SN}}$ and $\epsilon_{\mathrm{w}, \mathrm{AGN}}$ are free parameters representing the $\mathrm{SN}$ - and AGN-driven wind efficiencies, respectively. The adopted values are discussed in Section 3.

The ejection of metal-enriched gas and dust due to SN explosions and AGN activity enriches the IGM, increasing its metallicity and dust content. This leads to a corresponding increase in the initial gas metallicity and dust-to-gas mass ratio of DM halos collapsing at later times, affecting their star formation history, as well as the formation of nuclear black holes, as will be outlined in the next section.

\subsection{Black Hole formation and evolution}

Supermassive black holes are supposed to grow via both gas accretion and mergers starting from their seeds, less massive progenitors whose nature has a crucial role in understanding the origin of SMBHs. 


\subsubsection{Light BH Seeds}

In CAT the mass distribution of light BH seeds depends on the Pop III stellar initial mass function (IMF), that is still highly uncertain (Bromm 2013). According to the most recent numerical simulations of metal-free star forming regions hosted by minihalos at $z \sim 20-30$, the Pop III mass distribution ranges from a few $10 \mathrm{~s}$ to a few $100 \mathrm{~s} \mathrm{M}_{\odot}$ (Greif et al. 2011; Hirano et al. 2014; Stacy et al. 2016; Hosokawa et al. 2016; Sugimura et al. 2020). Following Valiante et al. (2016), we assume that Pop III stars form according to a Larson IMF:

$\Phi\left(m_{*}\right) \propto m_{*}^{\alpha-1} e^{-m_{*} / m_{\mathrm{ch}}}$

where $\alpha=-1.35, m_{\mathrm{ch}}=20 M_{\odot}$ and the possible range of stellar mass is $10 M_{\odot} \leq m_{*} \leq 300 M_{\odot}$. This choice is motivated by stellar archaeology studies and appears to best match the observed Galactic halo metallicity distribution function and the properties of $\mathrm{C}$-enhanced and $\mathrm{C}$-normal stars at $[\mathrm{Fe} / \mathrm{H}]<-3$ (de Bennassuti et al. 2014, 2017).

In our model, we stochastically sample the Pop III IMF untill we saturate the total stellar mass formed in each star formation episode. To consistently compute the $\mathrm{BH}$ remnants mass distribution, we assume that Pop III stars with masses in the range [40 - 140] $\mathrm{M}_{\odot}$ and [260 - 300] $M_{\odot}$ collapse directly to BHs of comparable mass (Heger \& Woosley 2002). Since these light BH seeds are expected to wander through the host galaxy, it is very unlikely that they will undergo mergers (Volonteri 2010), unless they form in binary systems (Sugimura et al. 2020). Moreover, dynamical effects like three-body scattering or gravitational recoil following $\mathrm{BH}$ mergers could lead to the ejection of the merging objects from the host galaxy (Campanelli et al. 2007), especially inside smaller dark matter halos (Dunn et al. 2020). For this reason, here we assume that only the most massive $\mathrm{BH}$ settles in the center of the galaxy and is considered as its light BH seed.

Pop III star formation can be sustained until the gas metallicity of the star forming region remains below a critical value $\mathrm{Z} \leq \mathrm{Z}_{\mathrm{cr}}$, where we assume $Z_{\mathrm{cr}}=10^{-3.8} \mathrm{Z}_{\odot}$ (Valiante et al. 2016). Above this threshold value, metal-fine structure lines and dust cooling increase the cooling efficiency (Omukai 2001; Schneider et al. 2002; Omukai et al. 2005; Schneider et al. 2006; Schneider et al. 2012), leading to a transition in the characteristic stellar masses. We therefore assume that above $\mathrm{Z}_{\mathrm{cr}}$, Pop II stars form in the mass range $0.1 M_{\odot} \leq$ $m_{*} \leq 100 M_{\odot}$ according to a Larson IMF with $m_{\mathrm{ch}}=0.35 M_{\odot}(\mathrm{de}$ Bennassuti et al. 2014, 2017).

\subsubsection{Heavy BH Seeds}

The second viable scenario for BH seed formation implemented in CAT is the so called Direct Collapse (DC) mechanism. Inside atomic-cooling halos (where $T_{\mathrm{vir}} \geq 10^{4} \mathrm{~K}$ ), where metal and dust cooling is still inefficient $\left(\mathrm{Z} \leq \mathrm{Z}_{\mathrm{cr}}\right)$, if the abundance of molecular hydrogen is suppressed by LW photons $(11.2-13.6 \mathrm{eV})$ inducing $\mathrm{H}_{2}$ photo-dissociation, the gas collapses almost iso-thermally with no fragmentation, leading to the formation of a single super massive star that becomes unstable, due to nuclear exhaustion or GR instabilities (Hosokawa et al. 2012; Inayoshi et al. 2014), and forms a heavy $B H$ seed, with mass in the range $\left[10^{4}-10^{6}\right] \mathrm{M}_{\odot}$ (Latif et al. 2013; Ferrara et al. 2014; Becerra et al. 2015; Latif \& Ferrara 2016; Becerra et al. 2018).

The importance of heavy BH seeds for the formation of high redshift SMBHs strongly depends on their birth rate that is still subject to large uncertainties (Inayoshi et al. 2020). If one neglects the effects of dynamical heating associated with structure forma- tion (Wise et al. 2019) or with major mergers (Mayer et al. 2010), the abundance of heavy BH seeds in the family tree of SMBHs depends on the adopted value of $Z_{c r}$ and on the critical value of the LW flux $\left(\mathrm{J}_{\mathrm{cr}}\right)$ above which $\mathrm{H}_{2}$ remains photo-dissociated. The latter condition is usually expressed as $\mathrm{J}_{\mathrm{LW}} \geq \mathrm{J}_{\mathrm{cr}}$, where $\mathrm{J}_{\mathrm{LW}}$ is the cumulative flux into the LW energy band in units of $10^{-21} \mathrm{erg} \mathrm{s}^{-1} \mathrm{~cm}^{-2} \mathrm{~Hz}^{-1} \mathrm{sr}^{-1}$. The value of $\mathrm{J}_{\mathrm{cr}}$ is still very uncertain and depends on (i) the total spectral energy distribution (SED) of the radiation background created by the various sources (Agarwal \& Khochfar 2015), (ii) the efficiency of $\mathrm{H}_{2}$ self-shielding, and (iii) the increase of the free electron fraction due to the presence of intense ionizing radiation which increases the $\mathrm{H}_{2}$ formation rate (Inayoshi \& Tanaka 2015). As a result, values of $\mathbf{J}_{\mathrm{cr}}$ ranging in a wide interval between $\sim 30$ and $\sim 10^{4}$ have been proposed in the literature (see Woods et al. 2019 and Inayoshi et al. 2020 and references therein). In addition, it has been recently suggested that the strong gas accretion rates may favour super-massive star formation event at higher metallicities than usually assumed (Chon \& Omukai 2020), through the so-called super-competitive accretion scenario. This has been shown to increase the number of heavy BH seeds by a factor ranging from 2 (Sassano et al. 2021) to 4 (Regan et al. 2020).

Following Valiante et al. (2016), here we adopt as threshold values $\mathrm{Z}_{\mathrm{cr}}=10^{-3.8} \mathrm{Z}_{\odot}$ and $\mathrm{J}_{\mathrm{cr}}=300$ to identify regions in atomiccooling halos where heavy $\mathrm{BH}$ seeds can form. If the conditions $\mathrm{Z}<\mathrm{Z}_{\mathrm{cr}}$ and $\mathrm{J}_{\mathrm{LW}} \geq \mathrm{J}_{\mathrm{cr}}$ are satisfied, we set in the center of the galaxy a heavy $B H$ seed with a mass of $10^{5} \mathrm{M}_{\odot}$.

\subsubsection{Black Hole Mergers}

Once formed, $\mathrm{BH}$ seeds are expected to grow via both gas accretion and coalescences with other BHs, eventually forming the SMBHs that power high redshift AGNs (see Volonteri 2010; Johnson \& Haardt 2016; Inayoshi et al. 2019, for complete reviews).

Following Valiante et al. (2011) we assume that two BHs coalesce only during major halo-halo mergers, i.e. if the mass ratio of their interacting host DM halos is $\mu>1 / 10$ (as defined in Section 2.1.2; Tanaka \& Haiman 2009). In our model, both the host galaxies and their nuclear BHs merge within the characteristic time interval of the simulation ( $\Delta t \sim 0.5-4 \mathrm{Myrs})$ and the merger product settles in the nuclear region of the final galaxy. Conversely, in minor mergers $(\mu<1 / 10)$, only the most massive $\mathrm{BH}$ is assumed to migrate in the center of the newly formed galaxy. The least massive one is instead considered as a satellite, wandering in the outskirts of the main galaxy (e.g. Callegari et al. 2009; Tamfal et al. 2018), and its subsequent evolution is no longer followed within the model.

Although oversimplified, our assumption is based on the common expectation that Keplerian BH binaries form promptly in interacting galaxies of "similar" mass and shrink to sub-pc separations (comparable to the primary $\mathrm{BH}$ influence radius) on relatively short timescales (within about a million years in the most optimistic cases; e.g. Mayer et al. 2007; Tanaka \& Haiman 2009). We will return to this point in Section 5.

\subsubsection{Black Hole Accretion}

Nuclear BHs are assumed to grow by accreting gas from the surrounding medium. The growth is regulated by the processes of star formation and mechanical feedback, which both lead to a depletion of gas inside the host galaxy. Following the original GQD model, in our reference model we assume that nuclear BHs accrete gas according to the Bondi-Hoyle-Lyttleton (BHL) accretion rate (Hoyle 
Table 1. Adopted set of parameters characterizing our cat reference model and the super-Edd and merger-driven model variants (see Section 3): the star formation efficiency $\epsilon_{\mathrm{SF}}$, the $\mathrm{SN}$ and AGN wind efficiencies $\epsilon_{\mathrm{w}, \mathrm{SN}}, \epsilon_{\mathrm{w}, \mathrm{AGN}}$ and the $\mathrm{BH}$ accretion parameter $\alpha$.

\begin{tabular}{lccccc}
\hline Model & $\epsilon_{\mathrm{SF}}$ & $\epsilon_{\mathrm{W}, \mathrm{SN}}$ & $\epsilon_{\mathrm{W}, \mathrm{AGN}}$ & $\alpha$ & $\epsilon_{\mathrm{r}}$ \\
\hline reference & 0.05 & $1.6 \times 10^{-3}$ & $2.5 \times 10^{-3}$ & 90 & 0.1 \\
super-Edd & 0.05 & $1.6 \times 10^{-3}$ & $2.5 \times 10^{-3}$ & 40 & Eq.(20) \\
merger-driven & 0.05 & $1.6 \times 10^{-3}$ & $2.5 \times 10^{-3}$ & 1 & Eq.(20) \\
\hline
\end{tabular}

\& Lyttleton 1941; Bondi 1952), given by:

$\dot{M}_{\mathrm{BHL}}=\alpha \frac{4 \pi G^{2} M_{\mathrm{BH}}^{2} \rho_{\mathrm{gas}}\left(r_{A}\right)}{c_{s}^{3}}$

In the above equation $c_{S}$ is the sound speed, which is estimated assuming a gas temperature $T_{\text {gas }}=T_{\text {vir }}$, and $\rho_{\text {gas }}\left(r_{A}\right)$ is the gas density evaluated at the Bondi radius, i.e. the radius of gravitational influence of the black hole, $r_{A}=2 G M_{\mathrm{BH}} / c_{s}^{2}$. Following Valiante et al. (2011), the gas density distribution is approximated as a singular isothermal sphere with a flat core:

$\rho_{\text {gas }}(r)=\frac{\rho_{\text {norm }}}{1+\left(r / r_{\text {core }}\right)^{2}}$

where $r_{\text {core }}=0.012 R_{\text {vir }}$ and $\rho_{\text {norm }}$ represents a normalization constant that ensures that, at each time step, the gas is distributed within the halo virial radius.

The parameter $\alpha$ in Eq. 12 does not appear in the original BHL formula and it is usually introduced in numerical simulation as a correction factor to take into account the enhanced gas density in the inner regions around the central $\mathrm{BH}$. In fact, due to the lack of resolution of the simulations, the actual BHL accretion rate tends to be strongly underestimated (Di Matteo et al. 2012; Schaye et al. 2015). As will be discussed in Section 3, the $\alpha$ parameter is a free parameter of the model ${ }^{8}$.

In our reference model, we assume that the $\mathrm{BH}$ accretion rate, $\dot{M}_{\mathrm{BH}}$, can not exceed the Eddington limit, so that:

$\dot{M}_{\mathrm{BH}}=\left(1-\epsilon_{r}\right) \dot{M}_{\mathrm{accr}}=\min \left(\dot{M}_{\mathrm{BHL}}, \dot{M}_{\mathrm{Edd}}\right)$,

where:

$\dot{M}_{\mathrm{Edd}}=\frac{L_{\mathrm{Edd}}}{\epsilon_{\mathrm{r}} c^{2}}$,

$\epsilon_{\mathrm{r}}$ is the radiative efficiency, i.e. the efficiency at which the accreting gas is converted into radiated luminosity,

$L_{\mathrm{Edd}}=\frac{4 \pi c G M_{\mathrm{BH}} m_{p}}{\sigma_{\mathrm{T}}}$

is the Eddington luminosity, $c$ is the speed of light, and $\sigma_{\mathrm{T}}$ is the Thomson cross-section. Here we assume that $\epsilon_{\mathrm{r}}=0.1$ (Shakura \& Sunyaev 1973), unless otherwise specified. The bolometric luminosity of the accreting $\mathrm{BH}$ can be expressed as:

$L_{\mathrm{bol}}=\epsilon_{\mathrm{r}} \dot{M}_{\mathrm{accr}} c^{2}$.

8 Note that since the Bondi radius is always much smaller than the core radius, the parameter $\alpha$ quantifies the unknown gas density enhancement around the black hole.

\subsection{Model variants}

As will be discussed in detail in Sections 4 and 5, our reference model, presented in the previous Section, shows the best agreement with different independent theoretical and observational constraints in reproducing the $\mathrm{BH}$ mass and luminosity distribution at $z \gtrsim 4$. However, we also decided to explore two additional model variants, in order to understand how the evolution of the AGN population depends on the assumed accretion paradigm. In fact, these alternative models do not change the $\mathrm{BH}$ seeding prescriptions but consider different scenarios for their mass growth, as described below.

\subsubsection{Exceeding the Eddington limit}

In the CAT reference model BHs are assumed to experience spherical accretion according to the Bondi-Hoyle-Lyttleton rate, with a maximum allowed limit at the Eddington rate (see 2.3.4). The BH mass dependence of both rates may represent a strong limitation to early $\mathrm{BH}$ growth, particularly in the case of light $\mathrm{BH}$ seeds.

Provided that the gas reservoir can be efficiently replenished, through large-scale accretion or galaxy mergers, $\mathrm{BH}$ seeds at high redshift may quickly reach accretion rates with Eddington ratios $\eta_{\text {Edd }} \equiv \dot{M}_{\mathrm{BHL}} / \dot{M}_{\text {Edd }} \rightarrow 1$. In order to check whether the restriction $\eta_{\text {Edd }} \leq 1$ provides a limitation to light $\mathrm{BH}$ seeds mass growth, we have explored an alternative model, that we dubbed super-Edd, where BHs are allowed to grow at super-Eddington rates (see Mayer 2019 and Inayoshi et al. 2020 for a thorough presentation of the main super-critical accretion models applied to $\mathrm{BH}$ seeds growth). Here we adopt the optically-thick, geometrically slim disk solution developed by Abramowicz et al. (1988), where part of the generated heat remains trapped within the accreting flow and is advected into the $\mathrm{BH}$, leading to a low radiative efficiency. In this model variant, BHs accrete gas according to the BHL formula (see Eq. 12), so that:

$\dot{M}_{\mathrm{BH}}=\dot{M}_{\mathrm{BHL}}$,

but their bolometric luminosity is computed according to the fitting formula for the radiative efficiency proposed by Madau et al. (2014) which, in turn, is based on the numerical solution obtained by Sądowski (2009):

$\frac{L_{\mathrm{bol}}}{L_{\mathrm{Edd}}}=A(a)\left[\frac{0.985}{\dot{M}_{\mathrm{Edd}} / \dot{M}_{\mathrm{accr}}+B(a)}+\frac{0.015}{\dot{M}_{\mathrm{Edd}} / \dot{M}_{\mathrm{accr}}+C(a)}\right]$.

where $A(a), B(a), C(a)$ are three functions of the black hole spin parameter $a=a_{\mathrm{BH}}$

$$
\begin{aligned}
& A(a)=(0.9663-0.9292 a)^{-0.5639} \\
& B(a)=(4.627-4.445 a)^{-0.5524}, \\
& C(a)=(827.3-718.1 a)^{-0.7060} .
\end{aligned}
$$

Note that in the above expression, the Eddington rate is defined as $\dot{M}_{\text {Edd }} \equiv 16 L_{\text {Edd }} / c^{2}$, i.e. it is a factor 1.6 larger than the definition given by Eq. (15). Hence, in this model variant, the radiative efficiency is computed as:

$\epsilon_{r}=\frac{L_{\mathrm{bol}}}{\dot{M}_{\mathrm{accr}} c^{2}}$.

To allow a better comparison with the reference model, the spin parameter has been assumed to be $a_{\mathrm{BH}}=0.572$ for all the BHs. 
This ensures that in the limit $\dot{M}_{\text {accr }}<<\dot{M}_{\text {Edd }}$ the radiative efficiency $\epsilon_{r} \rightarrow 0.1$, the same value adopted in the reference model.

With the above assumptions, large accretion rates up to $\dot{M}_{\text {accr }} \sim 100 \dot{M}_{\text {Edd }}$ lead to a radiated luminosity that remains only slightly super-Eddington, $L_{\text {bol }} \leq 5 L_{\text {Edd }}$. Therefore, this enables super-Eddington accretion while - at the same time - the effects of AGN feedback is still limited, reducing the energy injected in the surrounding medium, and favouring $\mathrm{BH}$ growth.

\subsubsection{Merger-driven black hole accretion}

As shown by Pezzulli et al. (2016) and Pezzulli et al. (2017b), super-Eddington accretion through a radiatively inefficient slim disk can be triggered by gas-rich galaxy mergers at high redshift. By implementing these prescriptions in GQD, it was found that - in highly biased regions, such as those that will later host bright quasars - episodic intense accretion is capable to grow $\sim 100 M_{\odot}$ light $\mathrm{BH}$ seeds to masses $\geq 10^{4} M_{\odot}$, comparable to the mass of heavy $\mathrm{BHs,}$ by $z \sim 20$. Since these biased regions are growing their mass at a faster pace, it is interesting to investigate the impact of this scenario on the general galaxy and AGN populations at $z \geq 4$.

Here we adopt a simplified description, that we refer to as merger-driven model, where we assume that, following a major merger, the gas inside the newborn galaxy is able to quickly loose angular momentum and the nuclear BH undergoes a period of enhanced accretion, with a rate given by:

$\dot{M}_{\mathrm{BH}}=\frac{\epsilon_{\mathrm{BH}} M_{\mathrm{gas}}}{\tau_{\mathrm{accr}}}$

where $M_{\mathrm{gas}}$ is the gas mass inside the newly formed galaxy, $\epsilon_{\mathrm{BH}}$ is the $\mathrm{BH}$ accretion efficiency and $\tau_{\mathrm{accr}}$ is the accretion timescale. We assumed an efficiency $\epsilon_{\mathrm{BH}}=1 / 3 \epsilon_{\mathrm{SF}}$ and a constant value of $\tau_{\mathrm{accr}}=10 \mathrm{Myr}$, that is comparable to the maximum dynamical timescale of the bulges found by Pezzulli et al. (2016) in their simulations. To be consistent with this merger-driven gas inflow scenario, the SFR in the newly formed galaxy is also enhanced by:

$\mathrm{SFR}=\frac{\epsilon_{\mathrm{SF}} M_{\mathrm{gas}}}{\tau_{\mathrm{dyn}, \mathrm{SF}}}$

where we assume a fixed star formation timescale $\tau_{\mathrm{dyn}, \mathrm{SF}}=\tau_{\mathrm{accr}}$. The enhancement in $\mathrm{BH}$ accretion and $\mathrm{SF}$ is assumed to terminate when either the ratio between the gas and $\mathrm{BH}$ masses, $M_{\mathrm{gas}} / M_{\mathrm{BH}}$, becomes lower than 10 or after a time interval $\Delta \mathrm{t}_{\text {burst }}=\tau_{\text {dyn }} / 100$. At that point, both star formation and $\mathrm{BH}$ accretion turn back to the quiescent mode, where the SFR is described by Eq. (6) and the $\mathrm{BH}$ accretion rate is described according to the $\mathrm{BHL}$ formula expressed by Eq. (12), but assuming $\alpha=1$ (see Table 1 for the set of assumptions and parameters characterizing this merger-driven growth model).

\section{MODEL CALIBRATION}

As discussed in Section 2 our model presents four parameters which are tuned to regulate the evolution of the baryonic component: the star formation efficiency $\left(\epsilon_{\mathrm{SF}}\right)$, the $\mathrm{SN}$ and AGN wind efficiencies $\left(\epsilon_{\mathrm{w}, \mathrm{SN}} ; \epsilon_{\mathrm{w}, \mathrm{AGN}}\right)$ and the $\mathrm{BH}$ accretion parameter $(\alpha)$. In Valiante et al. $(2011,2016)$ these free parameters were tuned to reproduce the SMBH mass and the properties of the host galaxy of SDSSJ1148+5251 at $z=6.4$ (Fan et al. 2002), a well known source, assumed to represent a prototypical example of luminous quasars at $z>6$. In this work we target the observed properties of high redshift quasars, as well as a realistic cosmic star formation history down to $z \geq 4$. Here we discuss the main observables used to calibrate the reference model, checking the consistency between predictions and observations. Although not reported, the same calibration has been performed for the model variants described in Section 2.4, see Table 1 for a summary of the adopted parameters.

\subsection{Cosmic star formation and stellar mass density}

We first tuned the model predictions on the observed total star formation rate density (SFRD), i.e. the total stellar mass formed per unit time and comoving volume. Measurements of the cosmic SFRD are mainly inferred from galaxy UV luminosity, currently limited to bright sources (UV magnitudes $M_{\mathrm{UV}} \leq-17.7$ ), which are also affected by dust extinction. To compare the model predictions with observations, we therefore consider the contribution from sources with UV luminosity above the observed threshold $\left(M_{\mathrm{UV}} \leq-17.7\right)^{9}$. To apply this luminosity cut, we convert the SFR of each galaxy to an intrinsic UV luminosity following the relation (Madau \& Dickinson 2014):

$L_{\mathrm{UV}}=\left(\frac{\mathrm{SFR}}{\mathrm{M}_{\odot} \mathrm{yr}^{-1}}\right) 7.14 \times 10^{27} \mathrm{erg} \mathrm{s}^{-1} \mathrm{~Hz}^{-1}$

Fig. 2 shows that the reference model (dashed red line) is in good agreement with the observed star formation rate density and stellar mass density evolution, from $z=4$ out to $z=8$. Note that CAT predicts a large number of currently undetected sources $\left(M_{\mathrm{UV}}>-17.7\right)$, which dominate the star formation rate density and stellar mass density at $z>4$ (solid red lines). The impact of dust extinction on the galaxy luminosity might increase even further the contribution coming from undetected sources, particularly at high redshift, where faint galaxies account for the majority of the total SFRD.

\subsection{Mass and luminosity of quasars at $z>5$}

In order to check whether our model, once calibrated, properly reproduces the properties of the observed population of high redshift quasars, here we compare the mass and bolometric luminosity of the most massive systems predicted by CAT at $z>5$ with the values inferred from quasar observations at similar redshifts. The results of the reference model at $z=5,6$ and 7 are shown, respectively, as yellow, orange, and dark red points in Fig. 3. The empty black data points show a sample including all $z>5.8$ quasars for which $\mathrm{BH}$ masses have been derived via MgII line single epoch virial estimator $^{10}$. The diagonal dotted lines indicate the Eddington luminosity as a function of the black hole mass.

We find that our simulated sample is composed mainly by lowmass objects, $\lesssim 10^{7.5} \mathrm{M}_{\odot}$, shining close to $0.1 \mathrm{~L}_{\text {Edd }}$ regardless of their redshift. More massive BHs show instead higher Eddington

9 The same correction is adopted to compare the cosmic stellar mass density (SMD) predicted by the model with observations.

10 We adopted the virial $\mathrm{BH}$ mass relation involving the $\mathrm{MgII}$ emission line full width half maximum and continuum luminosity at $3000 \AA$, using the calibration from Shen \& Liu (2012). The compilation of sources considered here has been assembled collecting data from the following works: Willott et al. (2010a); De Rosa et al. (2011); Wu et al. (2015); Mazzucchelli et al. (2017); Reed et al. (2017); Shao et al. (2017); Chehade et al. (2018); Eilers et al. (2018, 2020); Bañados et al. (2018); Wang et al. (2018, 2020); Shen et al. (2019); Reed et al. (2019); Onoue et al. (2019); Pons et al. (2019); Andika et al. (2020); Yang et al. (2020). 

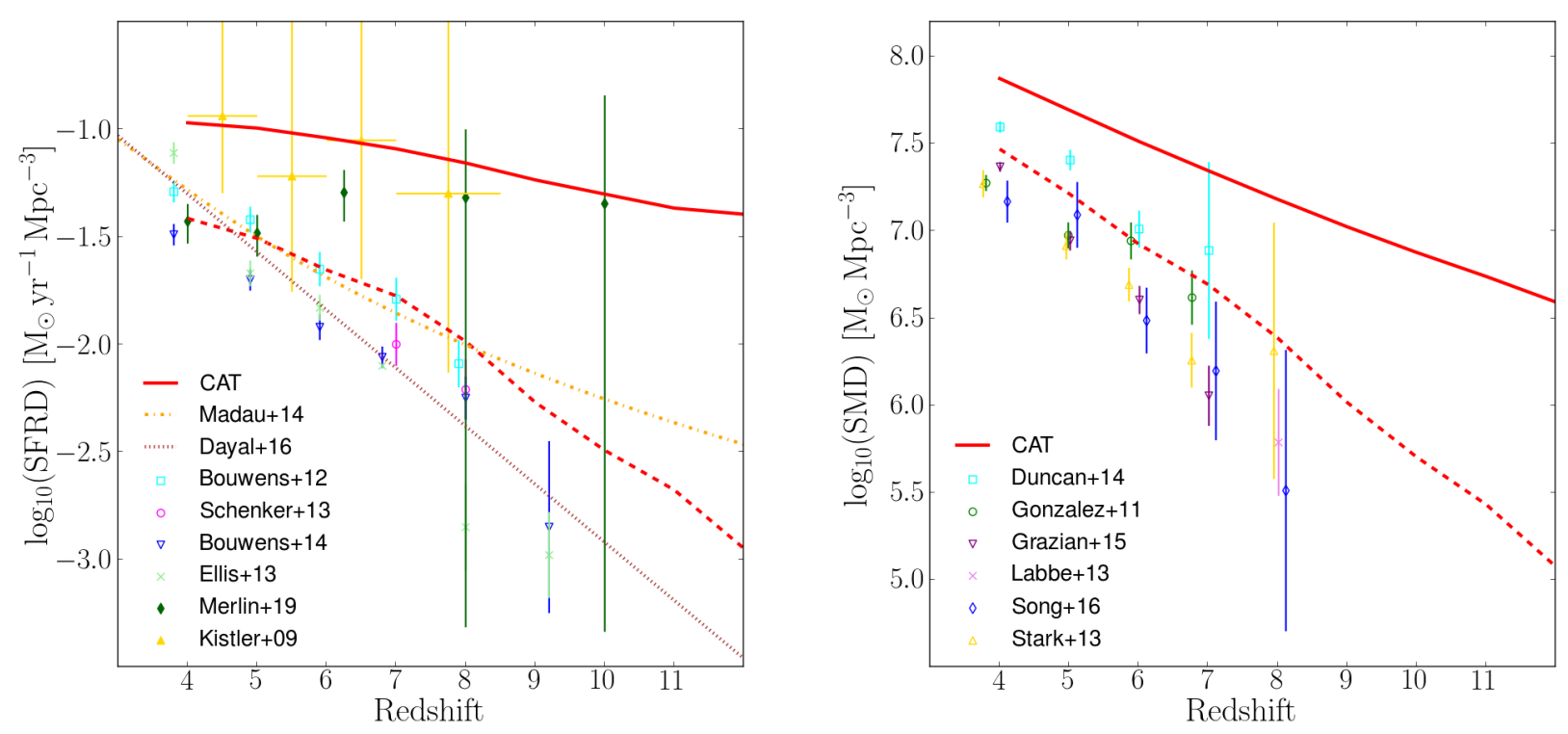

Figure 2. Redshift evolution of the comoving SFRD (left panel) and stellar mass density (right panel) predicted by CAT, when the entire galaxy population is considered (red solid lines) and when only galaxies with $M_{\mathrm{UV}}<-17.7$ are accounted for (red dashed lines). In each panel, the model predictions are compared with different observational results, as indicated in the legenda: Kistler et al. (2009), González et al. (2011), Bouwens et al. (2012), Labbé et al. (2013), Stark et al. (2013), Schenker et al. (2013), Ellis et al. (2013), Duncan et al. (2014), Bouwens et al. (2014), Oesch et al. (2014), Grazian et al. (2015), Song et al. (2016), Merlin et al. (2019). In the right panel, the yellow dashed-dotted line shows the empirical SFRD by Madau \& Dickinson (2014) while the brown dotted line shows the model prediction by Dayal et al. (2016).

ratios, especially at $z=6$ and 7 , where a large amount of gas is available for accretion. The comparison with observational data at $5.8<z<7.5$ (empty squares) shows that, despite our limited statistics due to the low number density of such objects, the most massive BHs predicted by CAT at similar redshifts populate the observed range of quasar masses and luminosities.

In conclusion, Figs. 2 and 3 show that the parameter values assumed in our reference model (see Table 1) guarantee a good agreement with the observed quasar population at $z>5$ and lead, at same time, to a galaxy population characterized by an evolution of the SFR density and stellar mass density in agreement with the available data.

Finally, a similar analysis has been carried out also for the two model variants, and the corresponding model parameters are reported in Table 1 .

\section{RESULTS}

The main motivation of our study is to investigate if, when and how the nature of $\mathrm{BH}$ seeds and their mass growth affect the population of BHs at $z>4$. In this Section, we first analyze the redshift evolution of the $\mathrm{BH}$ mass function predicted by the reference model and discuss how it changes in the two model variants. Second, we investigate whether the differences across models are revealed in the predicted luminosity functions, exploring at the same time their accordance with observational constraints obtained by current and future facilities.

\subsection{Black Hole Mass Function}

In Fig. 4 we show the BH mass function (MF) predicted by the reference model at different redshifts, ranging from $z=18$ down to $z=4$. Mass bins populated by light and heavy (i.e. at least with one DCBH progenitor) seed descendants are shown in magenta and red points, respectively. Here, as in the rest of the paper, binned data points are shown with the corresponding $1 \sigma$ Poisson error bars. The mass bin which includes newly formed and/or not grown heavy BH seeds has been highlighted in orange and it is not considered when fitting the distributions. In fact, the high number density of $10^{5} \mathrm{M}_{\odot}$ $\mathrm{BHs}$ reflects the adopted seeding prescription, while a more realistic heavy seed birth mass function (e.g. Ferrara et al. 2014) and/or the inclusion of the so-called medium-weight seed formation channel (Sassano et al. 2021) would probably result in a lower number density, spread across the intermediate mass range $\left(10^{3}-10^{5} \mathrm{M}_{\odot}\right)$.

It is immediately evident that the distribution is characterized by two well defined regions: $\mathrm{BH}$ descendants of light $\mathrm{BH}$ seeds, are confined below $10^{5} \mathrm{M}_{\odot}$, while $\mathrm{BHs}$ grown from heavy $\mathrm{BH}$ seeds dominate the high-mass end of the MF, i.e. above $10^{5} \mathrm{M}_{\odot}$. These two populations appear well separated across all the redshifts explored by our model. Early on $(z \geq 18)$ only light $\mathrm{BH}$ seeds are formed, some of which have already grown up to $\sim 10^{3.5} \mathrm{M}_{\odot}$. At $14 \leq z \leq 17$ the first heavy BH seeds start to form, but they do not significantly grow above their formation mass scale $\left(10^{5} \mathrm{M}_{\odot}\right)$. At these same redshifts, the distribution of light BH seed descendants has already reached its maximum extension in mass, well below $10^{4} \mathrm{M}_{\odot}$. At $z<14$ heavy $\mathrm{BH}$ seeds descendants continue to grow, and since their formation becomes progressively rarer, their mass distribution shifts to larger masses, covering the mass range $10^{6} M_{\odot}-10^{10} M_{\odot}$ at $z \lesssim 6$. Hence, the MFs of light and heavy 


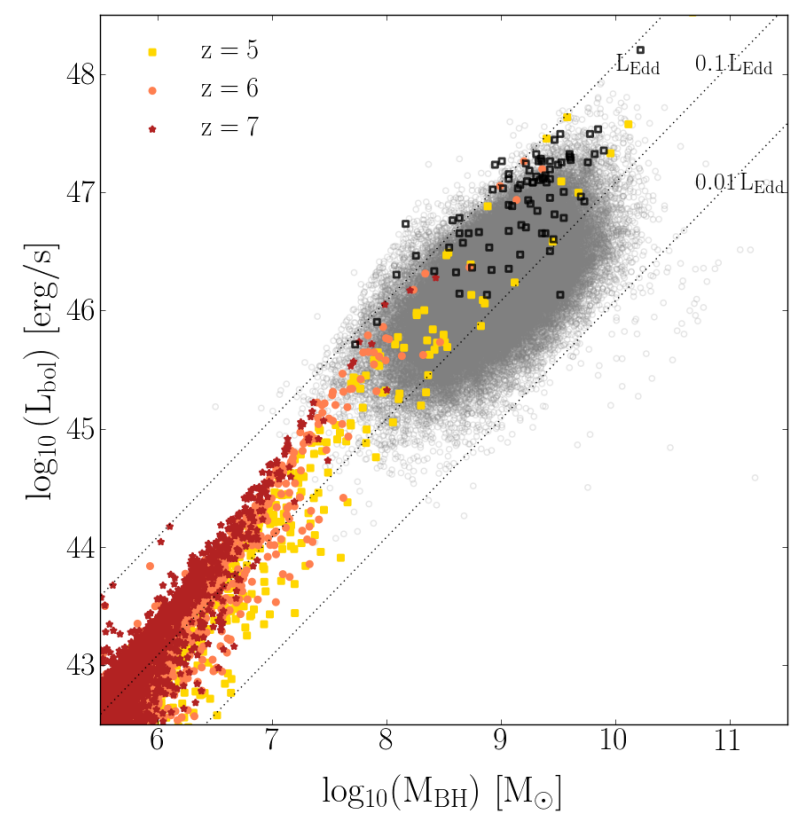

Figure 3. Bolometric luminosity as a function of the black hole mass for high redshift AGNs. Colored data points represent the quasar sample of the CAT reference model at $z=5$ (yellow), 6 (orange), and 7 (red). Black empty squares represent a collection of observed quasars at redshift $5.8<z<7.5$, while grey data show a large sample of quasars between $0.6<z<2$ drawn from the SDSS-DR7 quasar catalog by Shen et al. (2011) and for which MgII-based BH masses have been derived. Dotted lines marks the position of BHs with $L_{\text {bol }}=L_{\text {Edd }}, 0.1 L_{\text {Edd }}$ and $0.01 L_{\text {Edd }}$.

BH descendants remain completely segregated in mass as a consequence of the inefficient growth of light $\mathrm{BH}$ seeds. Some of these may be involved in major mergers contributing to the growth of the heavy seed descendants, others may instead participate in minor mergers, eventually becoming satellites (or wandering) BHs, which, in turn, explains why the highest mass bin of the distribution shifts to lower values with increasing time (decreasing redshift).

The gap in the $\mathrm{BH}$ number density just below the minimum adopted mass of heavy $\mathrm{BH}$ seeds indicates thus that in the CAT reference model light $\mathrm{BH}$ seeds fail to grow efficiently all the way down to $z \sim 4$. This could be a consequence of the host environmental properties of this class of seeds, namely the abundance of gas available to fuel $\mathrm{BH}$ growth, or the adopted mass accretion model. For this reason, we explored the redshift evolution of the BH MFs predicted by two model variants: the super-Edd model and the the merger-driven model (see Section 2.4 and Table 1 for more details).

Fig. 5 illustrates the results for the CAT super-Edd model. To aid the comparison with the reference model, the magenta and red dashed lines indicate the best fit to the MFs of light and heavy $\mathrm{BH}$ seeds predicted by the reference model (as in Fig.4). We find a significantly faster growth of heavy $\mathrm{BH}$ descendants, especially in the first phase after their formation, at redshifts $16 \lesssim z \lesssim 10$. At lower redshift this accelerated growth seems to slow down quickly and, despite the early build up of the global mass function, the maximum $\mathrm{BH}$ masses reached are $\sim 1$ dex below the results of the reference model. The rapid consumption of gas and the associated feedback in the early $\mathrm{BH}$ growth phase might be the reason for this behaviour. In fact, super Eddington accretion onto massive seeds leads to a quick depletion of gas from the host galaxy, which affects the subsequent BH evolution at later times. Hence, this super$E d d$ model well reproduces the global star formation history but struggles in reproducing the formation of the billion solar mass BHs observed to power quasars at $z>6$.

However, the super-Edd results show the same characteristic feature of the reference model observed in Fig. 4, i.e. a persisting gap between the light and heavy BH seeds distribution. This implies that the reason for the stunted growth of light $\mathrm{BH}$ seeds descendants does not reside in imposing an Eddington limit to the $\mathrm{BH}$ accretion. Instead, it could be due to a limited content of gas to fuel $\mathrm{BH}$ growth inside their hosts, or it could be a consequence of the adopted BHL accretion rate. In order to discern between these two possibilities, we investigate the predictions of the merger-driven model, shown in Fig. 6. In this figure we indicate again with magenta data points $\mathrm{BH}$ mass bins contributed only by light $\mathrm{BH}$ seeds descendants. However, for $\mathrm{BH}$ masses $\geq 10^{5} M_{\odot}$, the mass bins are now populated also by $\mathrm{BHs}$ grown from light $\mathrm{BH}$ seeds, in addition to those coming from heavy $\mathrm{BH}$ seeds progenitors and we show this mixed population in violet. In the previous reference and super-Edd models, instead, the high mass end of the $\mathrm{BH}$ mass function is entirely populated by $\mathrm{BHs}$ with at least one heavy $\mathrm{BH}$ seed progenitor. As a comparison, we also show with magenta and red dashed lines the best fit to the MFs of light and heavy BH seeds in the the reference model, respectively.

The merger-driven model shows a very rapid and early growth of light seeds, which reach $\mathrm{BH}$ masses as high as $10^{6} \mathrm{M}_{\odot}$ already at $z \sim 18$. The resulting distribution evolves shifting towards higher mass values, with minor changes in the global shape. This leads to a BH MF at $z \lesssim 6$ that continuously ranges between $\sim 10^{4}$ and $10^{10} \mathrm{M}_{\odot}$. At the low-mass end, the original gap has now disappeared since light $\mathrm{BH}$ seeds and their descendants can efficiently grow, competing with heavy seeds in SMBHs growth. The highmass end of the distribution, instead, reaches values consistent with the reference model, predicting however a slightly higher number density of objects between $\sim 10^{6}-10^{8} \mathrm{M}_{\odot}$.

It is important to note that the peak in the $\mathrm{BH}$ number density is shifted towards higher masses at later times (lower redshifts). This reflects the progressive depletion of low-mass objects as BH seeds are able to efficiently increase their masses through accretion and mergers, while newly formed seeds become rarer. The decreased number density of low-mass objects is clearly noticeable at $z \lesssim 6$.

In order to emphasize the contribution to the mass function of $\mathrm{BHs}$ grown only from light $\mathrm{BH}$ seeds in the merger-driven model, in Fig. 7 we show the mass function of heavy seed descendants at $z=4,6,8$ and 10 , along with their percentage of occupation of each $\mathrm{BH}$ mass bin. It is clear that, in this model variant, $\mathrm{BHs}$ descending only from light seeds represent a large fraction of the $\mathrm{BH}$ population. In particular, besides largely dominating the entire mass function at $z \gtrsim 10$, BHs grown from light seeds represent $\sim 50 \%$ of the SMBH population $\left(M_{\mathrm{BH}}>10^{5} M_{\odot}\right)$ down to redshift $z \sim 6$.

Hence, these results lead us to the conclusion that the main limiting factor to the growth of a $\mathrm{BH}$ seed is the mode of gas accretion rather than the gas mass reservoir around the black hole. The successful growth of light seeds predicted by the merger-driven scenario suggests that the key element affecting the efficiency of $\mathrm{BH}$ growth is the way in which the gas reaches the central regions, and therefore the physical mechanisms driving $\mathrm{BH}$ mass accretion.

However, we found that the large-scale mechanism of mergerdriven infall of gas toward the central regions around the nuclear BH is not sufficient, alone, to ensure an efficient growth of the smaller seeds. A black hole accretion model that allows super-Eddington 


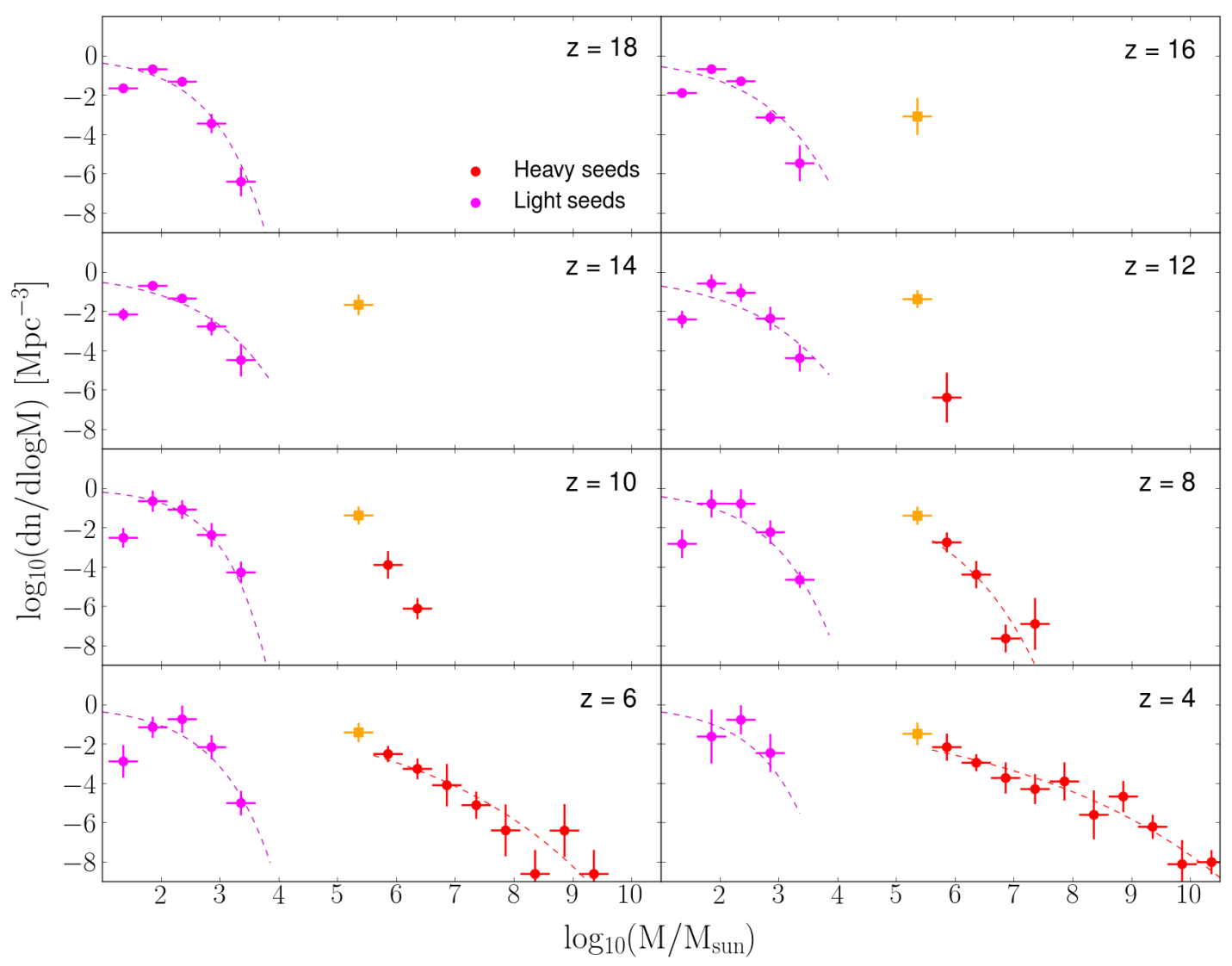

Figure 4. The BH MF predicted by the reference model at different redshifts, ranging from $z=18$ down to $z=4$. In each panel, we show the separate contributions of BHs descendants of light and heavy seeds with magenta and red points, respectively. The $1 \sigma$ Poisson error bars are shown in each data point. Where possible, the best fits of the two distributions are also shown with dashed lines. The orange data point represents the mass bin populated by newly formed and/or not grown heavy black holes seeds and it is not considered to produce best fit curves since its occupation is closely related to the adopted seed birth mass.

growth, as the slim disk model assumed in the merger-driven variant, is also needed to fill up the gap in the MF. In fact, assuming an Eddington limited growth in the merger-driven scenario, $\dot{M}_{\mathrm{BH}} \leq$ $\dot{M}_{\text {Edd }}$, we open-up again a clear gap in the mass distribution below $10^{5} \mathrm{M}_{\odot}$, similarly to that shown in Figure 5 and 6 . In addition, such a model predicts an overall reduction of the $\mathrm{BH}$ growth due to the low BH duty cycle assumed in the merger-driven scenario.

This suggests that the low-mass end of the BH mass function at $z \leq 6$ may provide important indications on the mass scale and growth rate of $\mathrm{BH}$ seeds at high redshift, while, at the high mass end, SMBHs with masses $\geq 10^{6} M_{\odot}$ are relatively insensitive to the nature of their $\mathrm{BH}$ progenitors. Still, the depopulation of the low-mass region (below $\sim 10^{5} \mathrm{M}_{\odot}$ ) predicted by the merger-driven model at $z \lesssim 6$ might resembles the clear gap in the MF predicted by a Bondi-like accretion mechanism, worsening the chances to clearly identify the distinctive features of different evolutionary scenarios.

The question is then whether current or future surveys may be able to discriminate among the various models, as it will be discussed in the next section.

\subsection{The AGN Luminosity Functions}

CAT model allows us to estimate the accretion rate of each nuclear black hole during its evolution through cosmic time, as determined by the environmental conditions present in its host galaxy. Hence, we are able to infer the luminosity of all active BHs (see Sections 2.3.4, 2.4.1, and 2.4.2) and to reconstruct their luminosity distribution, i.e. the AGN luminosity function (LF). Here we compare the bolometric LF predicted by CAT with several independent estimates based on multi-band observational data. These comparisons represent a fundamental benchmark for our $\mathrm{BH}$ evolution model, in order to determine which of the considered accretion scenarios better reproduce the observed trends. At the same time, they also allow us to check whether current or future surveys may be able to detect signatures of $\mathrm{BH}$ seeds populations and of their growth mode. A comparison between CAT predictions and the findings of the most recent AGN UV and X-ray surveys is discussed in Section 4.2.2 (see Figures 9, 10.) 


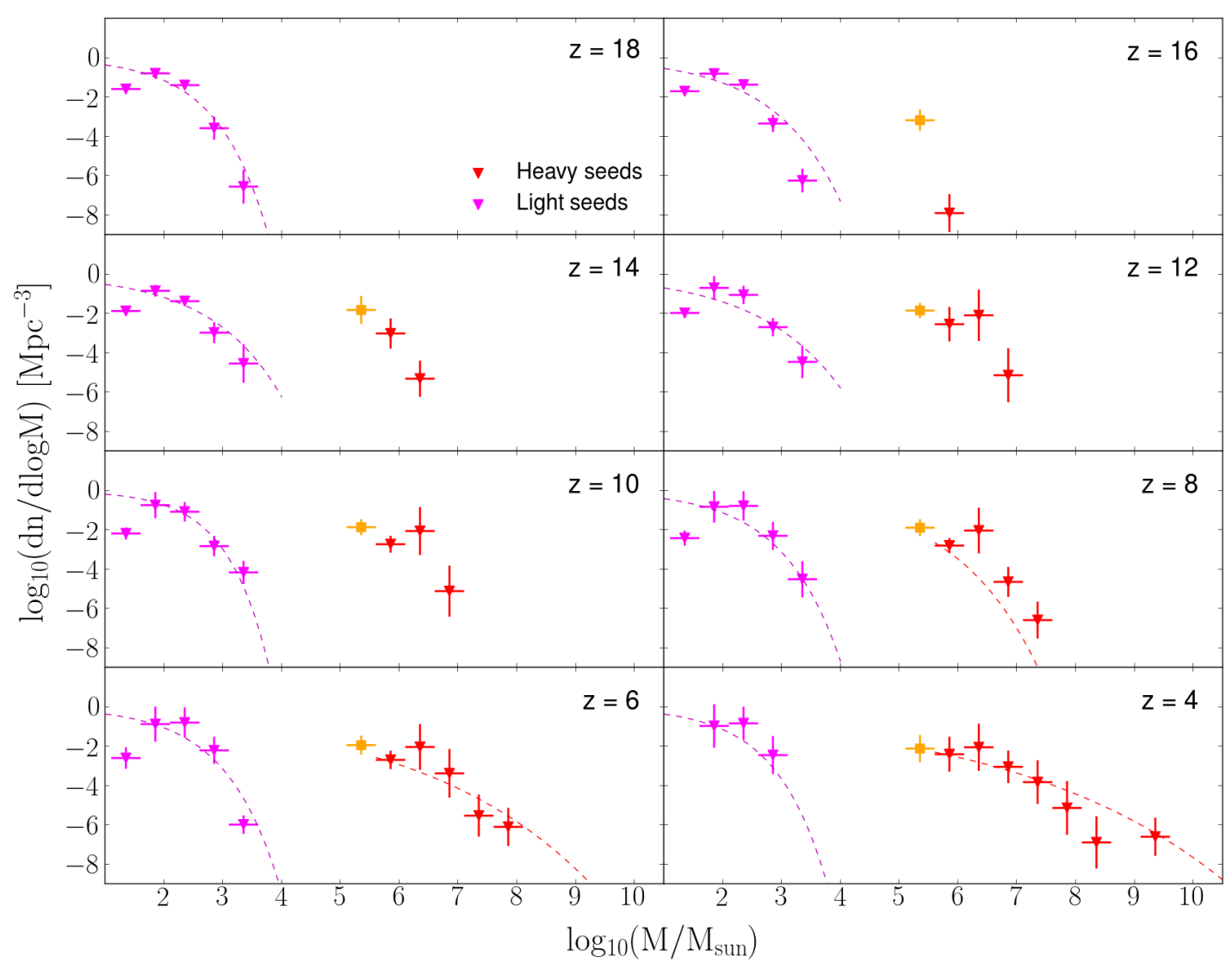

Figure 5. Same as Fig. 4 but for the super-Edd model (see Section 2.4.1). As a reference, we show with magenta and red dashed lines the best fits of the light and heavy $\mathrm{BH}$ seed descendant distributions obtained for the reference model.

\subsubsection{Bolometric Luminosity Function}

In Figure 8 we show the bolometric luminosity function predicted by all models in Table 1 at $z=4,5$ and 6 . In the reference and super$E d d$ models a clear gap in the luminosity function is visible below a threshold luminosity of $\sim 10^{41} \mathrm{erg} \mathrm{s}^{-1}$ at all redshifts. This reflects the behaviour of the $\mathrm{BH}$ mass functions shown in Figs. 4 and 5, and it is a consequence of the failed growth of light black hole seeds that characterize these two models, as previously discussed. While heavy seeds descendants continue to grow, populating the bright-end of the LF, the empty region in the luminosity distribution widens with decreasing redshift. In fact, as already described in Section 4.1, light seeds descendants are progressively more involved in galaxy and black hole merger events, eventually becoming satellites BHs or merging with heavy descendants.

In the merger-driven model instead the efficient growth of light $\mathrm{BH}$ seeds leads to a continuous luminosity distribution at all redshifts. However, at $z \sim 6$ the mass distribution shown in Fig. 6 results in a LF which peaks around $\sim 10^{40-42} \mathrm{erg} \mathrm{s}^{-1}$ and then quickly drops down at fainter luminosities. This behaviour, as mentioned before, reflects the progressively lower number of lighter seeds, due to both the involvement in galaxy mergers or their efficient growth toward higher masses.

Note that this third model is characterized by brief periods of enhanced accretion onto BHs. If we infer the LF at a single given time, thus, we might under-sample rare objects with high luminosity that are active only for a short interval of time. Therefore, following Griffin et al. (2019), when computing the LF for the merger-driven model we average the LF over a time window $\Delta t_{w}$ of $\sim 50 \mathrm{Myr}$ around each redshift of interest. To reconstruct the global luminosity distribution, each active object is then assigned a weight

$w=t_{\mathrm{Q}} / \Delta t_{\mathrm{W}}$,

where $t_{\mathrm{Q}}$ is the time spent in the enhanced accretion mode during the considered time window.

The model predictions are compared to the results obtained by Willott et al. (2010b), based on SDSS and CFHQS data, and with the LF evolutionary models proposed by Shankar et al. (2009) and Hopkins et al. (2007). We also show the LF constraints presented by Shen et al. (2020), which are based on a large compilation of AGNs observations in different energy bands, from the rest-frame IR to the $\mathrm{X}$-rays. Finally, we compare our findings with the results of the Xray studies by Ueda et al. (2014), applying the bolometric correction proposed by Duras et al. (2020). The shaded region shows the range of uncertainties associated to obscuration effects, with the upper 


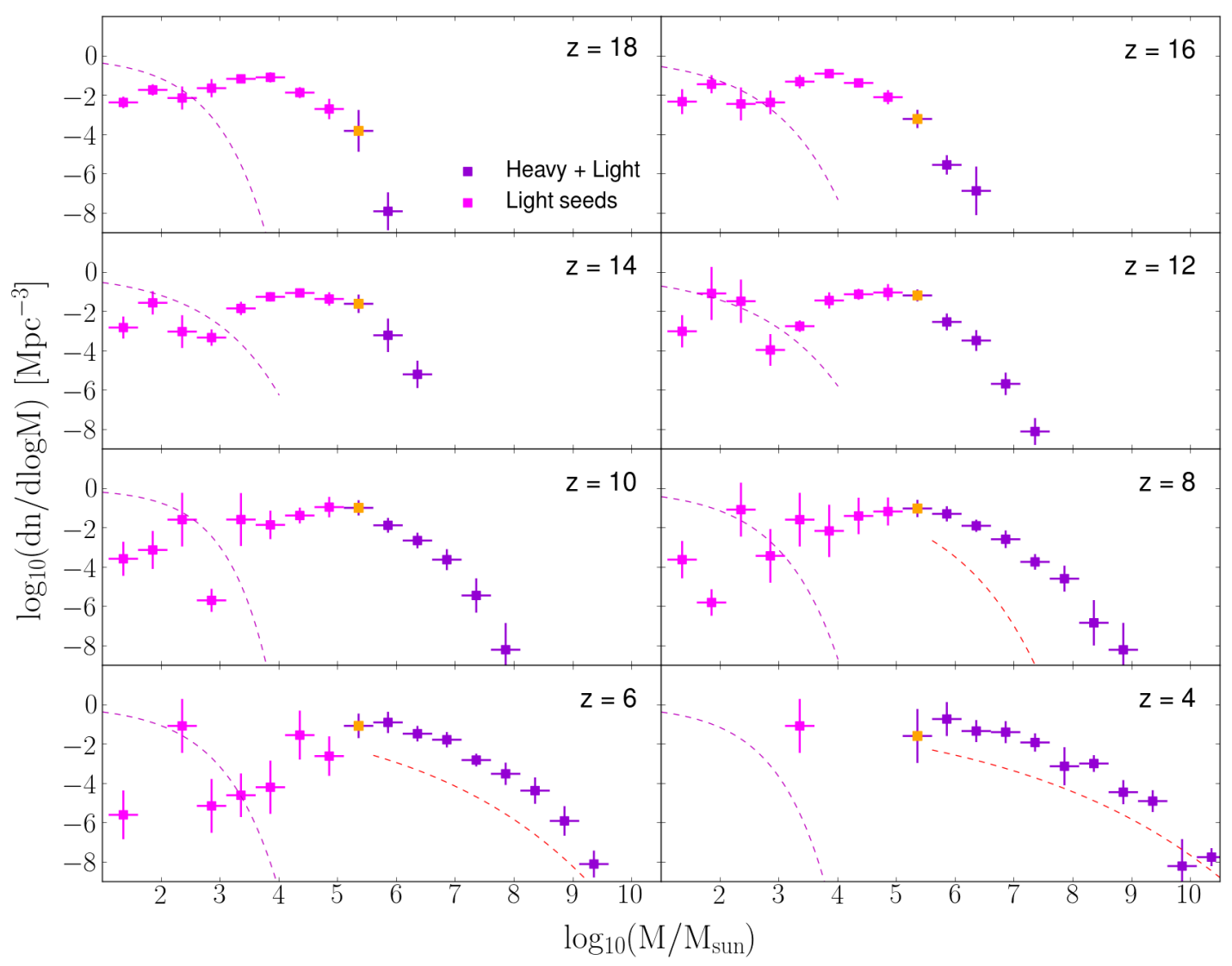

Figure 6. Same as Fig.5 but for the merger-driven accretion model (see Section 2.4.2). Here we show with violet data points the mass bins above $10^{5} \mathrm{M}_{\odot}$ since, unlike the previous models, they are now populated by both light and heavy BH seed descendants. Distributions (data points with error bars) are compared with best fits obtained for the reference model (dashed lines).

and lower bounds illustrating the LF obtained with and without obscuration correction, respectively. ${ }^{11}$

The LFs predicted by the reference model are remarkably consistent with the observed bright-end distributions $\left(\gtrsim 10^{42} \mathrm{erg} \mathrm{s}^{-1}\right.$ ) at all the considered redshift, $z=4-6$. The number of sources is instead slightly over-predicted at fainter luminosities, $\sim 10^{40-42} \mathrm{erg} \mathrm{s}^{-1}$, where, however, the observations should be largely affected by obscuration effects.

The distribution of low-luminosity sources predicted by the super-Edd model (middle panels of Figure 8) is similar to that described in the reference case. Conversely, the number density of brighter sources, above $\sim 10^{44} \mathrm{erg} \mathrm{s}^{-1}$, dramatically drops, especially at $z=5$ and 6 , as a consequence of the lack of massive/luminous AGN predicted in the model.

This result suggests that the accelerated growth of BH seeds at early

11 Here the obscuration correction has been applied assuming a fraction of Compton-thick AGNs with column density $24<\mathrm{N}_{\mathrm{H}}<26$ four times higher that the one considered in Ueda et al. (2014). This is in agreement with the recent work of Ananna et al. (2019), which found a much higher number density of Compton-thick objects with respect to the original work of Ueda et al. (2014). times, driven by the super-Eddington accretion (see Fig. 5), strongly affects the $\mathrm{BH}$ evolution at later times. In particular, the enhanced accretion rate and the associated feedback significantly deplete the gas reservoir available around the BH. On the other hand, new infalling material from the external medium is mainly consumed by star formation that is quite efficient in massive halos hosting growing heavy seeds. Hence, the growth in mass of the initial seed remains shortly stuck and it will undergo major accretion episodes only as a consequence of gas-rich mergers, failing to reproduce both masses and luminosities of the extreme quasars observed at $z \sim 6$.

In the merger-driven model (right panels of Figure 8) the bright-end of the luminosity function $\left(L_{b o l} \gtrsim 10^{46} \mathrm{erg} \mathrm{s}^{-1}\right)$ is consistent with the observational data at $4 \leq z \leq 5$. At $z=6$ the LF is instead over-estimated as a consequence of the early mass growth of BHs driven by halo mergers. At the faint-end of the distribution, the merger-driven model over-predicts the number of objects at all redshift within the $10^{41} \mathrm{erg} \mathrm{s}^{-1} \lesssim \mathrm{L}_{\text {bol }} \lesssim 10^{45} \mathrm{erg} \mathrm{s}^{-1}$ luminosity range. Compared to the reference and super-Edd models, this is due to the population of efficiently grown light seed descendants that were unable to significantly grow in mass in the classic Bondi-like accretion scenario. At even fainter luminosities, below $\sim 10^{40} \mathrm{erg} \mathrm{s}^{-1}$, the LF drops down similarly to what we observe in 


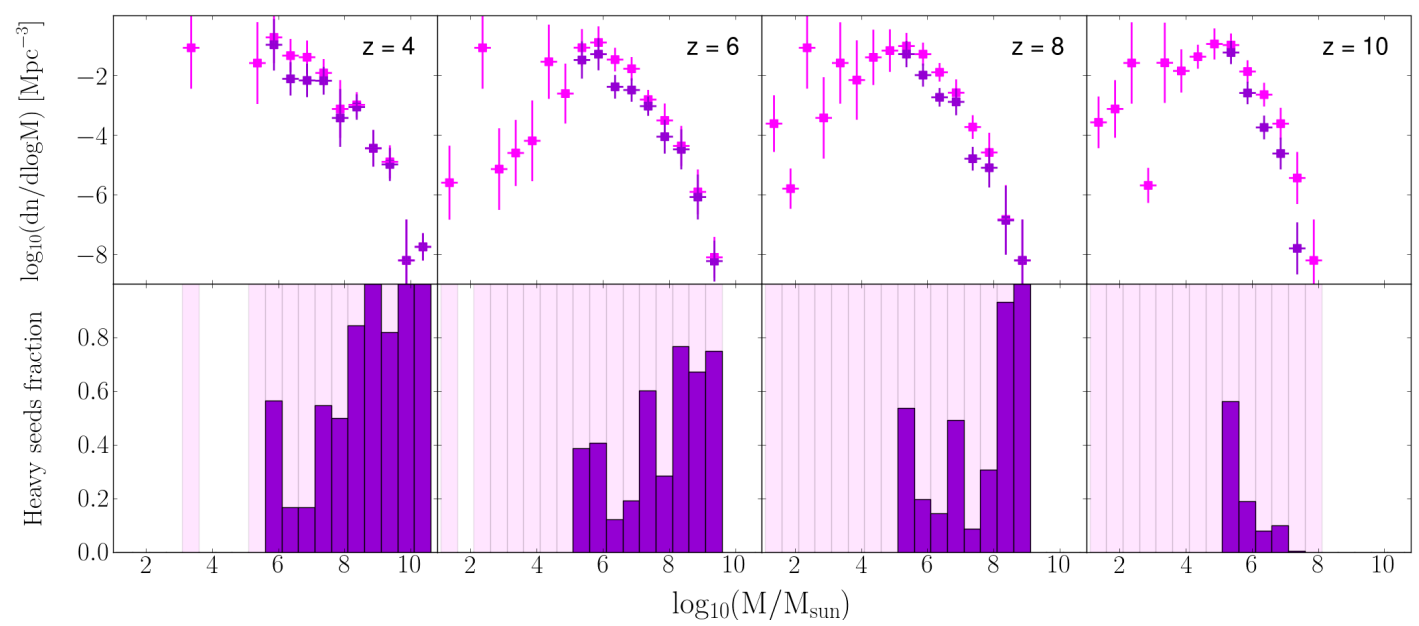

Figure 7. The Figure shows, for the merger-driven model, the relative contribution to the final BH mass function of BHs descending from at least one heavy or only light $\mathrm{BH}$ seeds. Top row: the total BH mass function (magenta points, same as in Fig. 6) and the contribution of only heavy-BH seeds descendants (violet) at $z=4,6,8$, and 10 (from left to right). Bottom row: the corresponding percentage of heavy BH seed descendants in different mass bins. BHs descending only from light-BH seeds (represented by lighter magenta regions of the histograms) clearly provide, in this model, a significant contribution to the global MF, even at the high-mass end.

the reference model, but as a result of the exact opposite process. In fact, while in the BHL accretion scenario the lack of sources below this threshold is due to the low mass of light seed descendants, here most of them grows efficiently, depopulating this luminosity region at low redshift.

It is worth considering that if the bursts of accretion driving BH growth take place on very short timescales, $\lesssim 2$ Myrs, the $t_{Q}$ parameter in Eq. 24 will be over-estimated, explaining, at least partially, the higher number density of sources predicted by the merger-driven model compared to observations, as will be highlighted in detail in Section 4.2.2.

Note that, for the merger-driven scenario, we considered a simplified version of the original model proposed by Pezzulli et al. (2016) mainly to investigate the impact of the accretion model on the overall shape of the $\mathrm{BH}$ luminosity and mass distributions.

A more sophisticated description of $\mathrm{BH}$ growth triggered by gas-rich galaxy mergers will be developed in future works to further explore its comparison to observations.

It is important to emphasize that the agreement of the reference model with observations of the bright-end of the LF is an important result, as it implies that CAT is capable to reproduce reliable mass and luminosity distributions of the population of massive $\left(\gtrsim 10^{7} M_{\odot}\right)$ nuclear BHs down to $z \sim 4$, despite the $\mathrm{BH}$ seeding and growth model was originally aimed at reproducing the evolution of the most extreme SMBHs $\left(\sim 10^{9} \mathrm{M}_{\odot}\right)$ at $z \geq 6$. The above result suggests that the mechanisms which drive the formation and evolution of these class of massive BHs across cosmic times remain the same, regardless of the mass of the final object and of its host galaxy.

However, as already observed, if we extrapolate the empirical $\mathrm{LF}$ at luminosities $\lesssim 10^{42} \mathrm{erg} \mathrm{s}^{-1}$, the reference model seems to predict an excess of faint AGNs compared to observations. This may be a hint that the accretion mechanism assumed in CAT leads to an overgrowth of less massive BHs. In order to properly reproduce the faint end of the BH LF, in fact, similar semi-analytic models artificially shut off $\mathrm{BH}$ growth below a given halo mass (e.g. Piana et al. 2021), assuming that this is caused by the impact of SN feedback (Habouzit et al. 2017; Anglés-Alcázar et al. 2017). Hence, an improved treatment of the accretion process of less massive nuclear BHs may be required to provide a better agreement with the empirical relations at low luminosities, as will be discussed in Section 5.

Nevertheless, we should note that the empirical LF predicted at $z \gtrsim 4$ are constrained mainly by luminous sources with $\mathrm{L}_{\text {bol }} \gtrsim 10^{43} \mathrm{erg} \mathrm{s}^{-1}$. The distributions at lower luminosities are thus highly uncertain, especially since the population of fainter AGNs might be heavily obscured, as suggested by several studies (Matsuoka et al. 2018; Giallongo et al. 2019). The improved agreement at faint luminosities observed in the UV and X-ray LFs between CAT predictions and several observations (see Section 4.2.2) supports the idea that these tensions might be due, at least partially, to observational limits.

Overall, this comparison suggests that the reference model provides the closest match to current observations, which however are able to probe only the bright-end of the predicted LF, dominated by heavy $\mathrm{BH}$ seeds descendants. Yet, at the very faint-end, below the gap, the LF is due to the more numerous population of inefficiently growing light $\mathrm{BH}$ seeds which remains completely invisible, as they lie at luminosities that are 4 orders of magnitudes lower than the faintest luminosity probed by current data. We also find that a significantly different evolutionary scenario, such as the mergerdriven model, might lead to comparable results for the bright-end of the AGN luminosity distribution. The main differences show up instead in the low luminosity region, making therefore very challenging to discern between different accretion models through observational campaigns.

A more detailed comparison of the model predictions with current and forthcoming observational facilities, in particular with the most promising deep sky surveys, is carried out in Section 4.2.2.

The improved sensitivity of the forthcoming generation of surveys might nevertheless push down the observational limit toward the threshold of $10^{41} \mathrm{erg} \mathrm{s}^{-1}$, which is less than two orders of magnitude below the actual empirical constraints. In this range of lumi- 


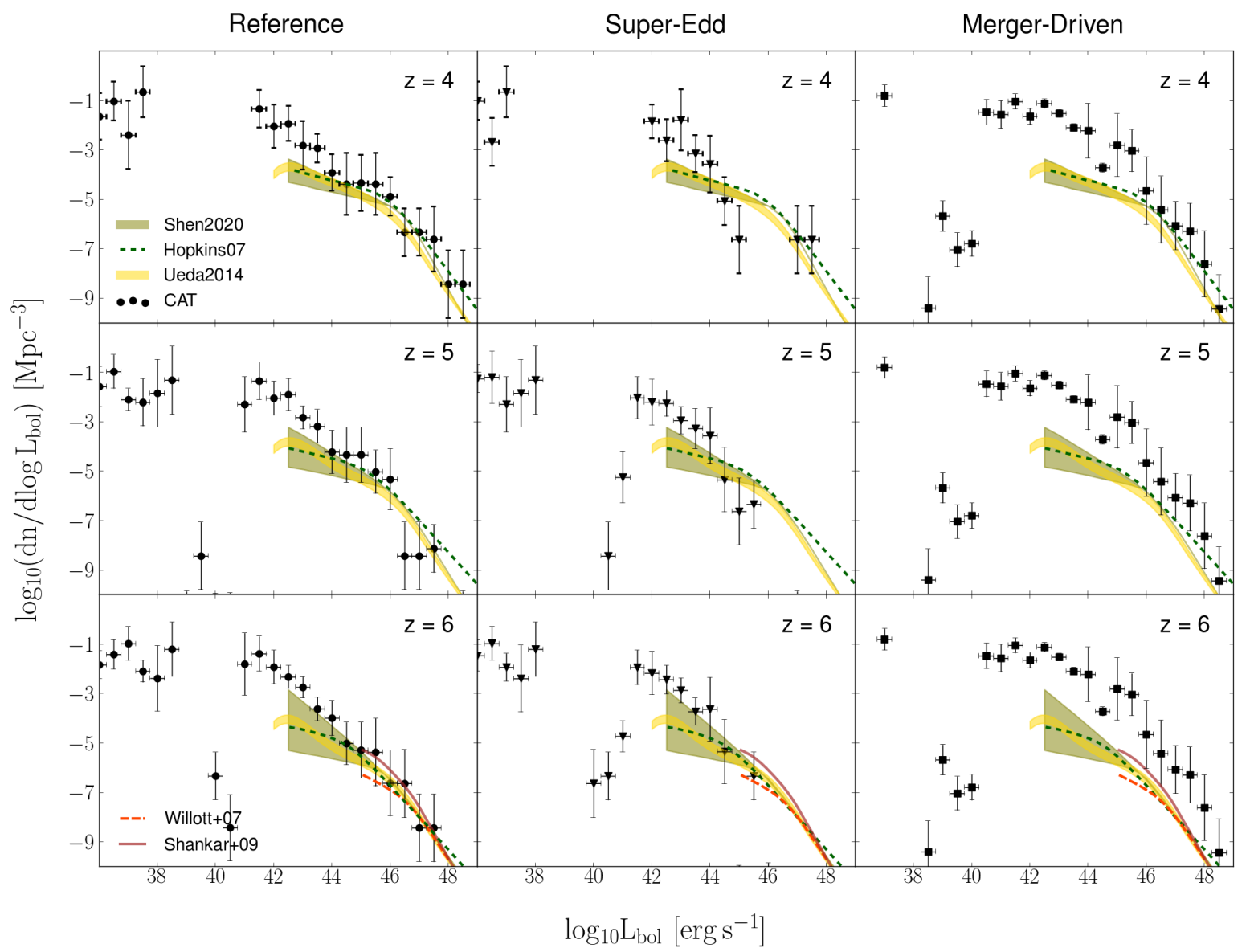

Figure 8. Bolometric luminosity function of AGNs at $z=4,5$ and 6 (from top to bottom). Black data points show the luminosity distribution of cat AGN population. Each column shows the results obtained in one of the three models examined: the reference model (left panels), the super-Edd model (central panels) and the merger-driven model (right panels). Our results are compared with the predictions of Hopkins et al. (2007, green dashed lines), Willott et al. (2010b, red dashed line), Shankar et al. (2009, brown solid line) and Shen et al. (2020, green shaded area). We also show as a comparison the constraints on the bolometric LF derived from the X-ray data by Ueda et al. (2014) (yellow shaded area, see text).

nosity the number density of accreting BHs is maximally sensitive to the assumed model for black hole growth, as shown in Fig. 8, despite host contamination might become significant for such faint sources. Still, even at these luminosities, different scenarios for black hole fueling and accretion might result in similar luminosity distributions, characterized by a decreasing number of sources below $\sim 10^{40} \mathrm{erg} \mathrm{s}^{-1}$.

\subsubsection{BH UV and X-ray Luminosity Function}

In order to compare more extensively cAT predictions with current and future observational facilities, and in particular with the most promising deep sky surveys, we derived, for the three model variants, the $\mathrm{BH}$ luminosity distribution predicted in the UV and X-ray energy bands. The bolometric luminosity of each active BH can be converted into a B-band and X-ray luminosity adopting specific bolometric corrections, such as in Duras et al. (2020):

$\frac{L_{\mathrm{bol}}}{L_{\mathrm{B}}}=5.13 \quad ; \quad \frac{L_{\mathrm{bol}}}{L_{\mathrm{X}}}=a\left[1+\left(\frac{\log \left(L_{\mathrm{bol}} / L_{\odot}\right)}{b}\right)^{c}\right]$

where $L_{\mathrm{B}}$ and $L_{\mathrm{X}}$ are, respectively, the luminosity in the B (4400 $\AA$ ) and (2-10) keV band, while $a=10.96, b=11.93$ and $c=17.79$ are parameters calibrated on a population of both type 1 and type 2 quasars. Then, the B-band luminosity has been converted into a UV luminosity at $1450 \AA$ assuming that $L_{v} \propto v^{-0.44}$ (Dayal et al. 2019).

However, for a proper comparison with observational data we must account for obscuration effects. In fact, the intrinsic LFs predicted by CAT have to be corrected for the fraction of obscured AGNs in each energy band. Following Merloni et al. (2014), we assume the fraction of obscured AGNs in the UV band to be a decreasing function of the intrinsic X-ray luminosity of the source. Hence, we 
compute the observable fraction as:

$f_{\text {obs }}=1-0.56-\frac{1}{\pi} \arctan \left(\frac{43.89-\log \mathrm{L}_{\mathrm{X}}}{0.46}\right)$

We show the resulting LFs, that we call obscured AGN UV LFs, in Fig. 9. Here, the predictions obtained for the three different models at redshift $z=4,5$ and 6 are represented by the black data points with error bars. The black long-dashed lines represent the best-fit to the distributions at each redshift, which are well represented by broken power-laws that flatten below a characteristic luminosity. For comparison, in each panel we also show the corresponding galaxy UV LFs predicted by CAT model at the same redshift. In order to take into account dust extinction, we corrected the galaxy LFs as

$L_{\mathrm{UV}, \mathrm{obs}}=L_{\mathrm{UV}} \exp \left[-\Sigma_{\text {gas }} \mathcal{D} \mathrm{k}_{\mathrm{UV}}\right]$

where $\Sigma_{\text {gas }}$ is the gas surface density, $\mathcal{D}$ the dust-to-gas mass ratio and $k_{\mathrm{UV}}$ is the extinction coefficient per unit mass in the energy band of interest. The value of $k_{\mathrm{UV}}$ has been inferred considering the extinction curve of the Small Magellanic Cloud (SMC) (Weingartner \& Draine 2001). We assumed here a simple screen model where the optical depth is computed considering the contribution of all the gas and dust mass inside the galaxy. This will possibly result in an overestimation of the impact of dust obscuration, if compared to more sophisticated two-phase dust extinction models (see e.g. Mancini et al. 2016). Therefore, in Fig. 9, we show as a grey shaded area the region enclosed between the best fit of the intrinsic and dust-corrected galaxy UV LFs. In the reference and super-Edd models, we observe that dust extinction might heavily affect the galaxy UV luminosity. In the merger-driven scenario, instead, the lower abundance of gas due to the more competitive mechanisms of star formation and $\mathrm{BH}$ accretion translates into a smaller difference between the intrinsic and dust-corrected luminosity functions.

However, for all the models and at all redshifts, the UV LF of AGNs dominates at the bright-end, i.e. for magnitudes $M_{1450} \leq$ -22 . At fainter luminosities, up to $M_{1450} \sim-20$, the AGN LF still dominates only if the UV emission from the host galaxy is heavily reduced by dust extinction. Finally, at even fainter magnitudes the UV LF is dominated by the stellar emission from the host galaxies.

In the same figure, CAT model predictions are compared to the observations by the SHELLQs survey (Matsuoka et al. 2018), the CFHT Legacy Survey (McGreer et al. 2018), and the Hyper Suprime-Cam Wide Survey (Akiyama et al. 2018; Niida et al. 2020) (blue triangles and green diamonds). The figure also shows the results of the analysis by Parsa et al. (2018) and Giallongo et al. (2019), who estimated the AGN UV LF using X-ray data, resulting in a LF which should be less affected by obscuration. We also report the results of the COSMOS spectroscopic survey conducted by Boutsia et al. (2018).

Among the three models, the reference one provides the best agreement with observational constraints. In particular, at fainter magnitudes the predicted LF well trace the observations of Giallongo et al. (2019), Parsa et al. (2018) and Boutsia et al. (2018). In the bright end of the LF, instead, the distribution predicted by the reference model shows a more pronounced scatter due to the lower statistics, especially at $z \sim 6$. However, if we fit the obtained data with a broken power-law, we find again a close agreement with the empirical data, despite a slight over-prediction in the number density of bright sources at $z=4$.

The agreement with the data is worse for the super-Edd model, which in fact, despite obtaining results similar to the reference model at $M_{\mathrm{UV}} \gtrsim-19$, fails to reproduce the observed distributions at higher luminosities as pointed out also for the bolometric LF (Section 4.2).

Lastly, the merger-driven model shows an overall distribution very similar to that predicted by the reference model but shifted towards higher luminosities, which results in a higher number of sources at bright magnitudes $M_{1450} \lesssim-23$ compared to the observed one.

Interestingly we observe that at lower luminosities in all the three model variants the predicted LFs appear to be in better agreement with the LFs obtained through X-ray selection techniques. This suggests, as already outlined by Matsuoka et al. (2018) and Giallongo et al. (2019), that the apparent tension between different observational results might be due to an increasing incompleteness at fainter magnitudes or to an higher fraction of obscured AGNs toward lower luminosities.

It is important to note that in none of the models the UV LFs show the clear gap that was present in the bolometric LFs. This is because in the reference and super-Edd models, the gap appears at UV magnitudes $M_{1450} \geq-10$, that are several orders of magnitudes below the sensitivity limits of current observational facilities. Even with the sensitivity of JWST, whose luminosity limit is shown with vertical dashed lines in Fig. 9 (Griffin et al. 2020), it will be impossible to observe such faint sources, unless with the help of gravitational lensing. In addition, at magnitudes $M_{1450} \gtrsim-22$ deblending techniques need to be applied to discriminate the AGN emission and the emission coming from star formation in the host galaxy.

Using Eq. 25, we have also computed the LFs predicted by the three CAT models in the [2-10] keV X-ray energy band. The results are shown in Fig.10. Black data points and error bars represent the binned intrinsic LFs at redshift $z=4,5$ and 6 (from top to bottom) and different columns refer to the three CAT model variants that we have considered (reference, super-Edd, and merger-driven models from left to right). In each panel, the dashed lines represent the best-fit to the model predictions and the dotted lines show the Xray LFs contributed by star formation in the host galaxies at the same redshift. The latter component has been estimated using the empirical relation proposed by Fornasini et al. (2018):

$L_{\mathrm{XRBs}}=10^{29.98}(1+z)^{0.62} M_{*}+10^{39.78}(1+z)^{0.2} \mathrm{SFR}^{0.84}$

where $M_{*}$ and SFR are, respectively, the galaxy stellar mass in solar units and the star formation rate in solar masses per year. In Fig. 10 CAT predictions are compared with the X-ray LFs obtained by Fiore et al. (2012), Ueda et al. (2014), and Miyaji et al. (2015), which are some of the most complete studies of the AGN X-ray emission up to redshift $z \sim 4-6$. We also show the LF obtained by Vito et al. (2018) through a wide sample of AGNs at redshift $3.6>z>6$.

Since each of these works accounts differently for the fraction of absorbed and obscured AGNs, we decided to show with a shaded region, for the reference and the merger-driven models, how the bestfit distribution of the CAT XLF changes considering absorption for the Compton-thin AGN population. We assume here the fraction of un-absorbed quasars $\psi_{\mathrm{X}}$,unabs as a function of the X-ray luminosity as proposed by Ueda et al. (2014):

$\psi_{\mathrm{X}, \text { unabs }}=1-\min \left[\psi_{\max }, \max \left[\psi^{*}-\beta\left(\log \mathrm{L}_{\mathrm{X}}-43.75\right), \psi_{\min }\right]\right]$

where $\psi_{\max }=0.84, \psi_{\min }=0.2, \psi^{*} \simeq 0.73$ and $L_{X}$ is the [2$10] \mathrm{keV}$ luminosity in $\mathrm{erg} \mathrm{s}^{-1}$. It important to note, however, that the distributions corrected for quasar absorption represent only an upper limit for the un-obscured AGN XLF, since we do not consider the 


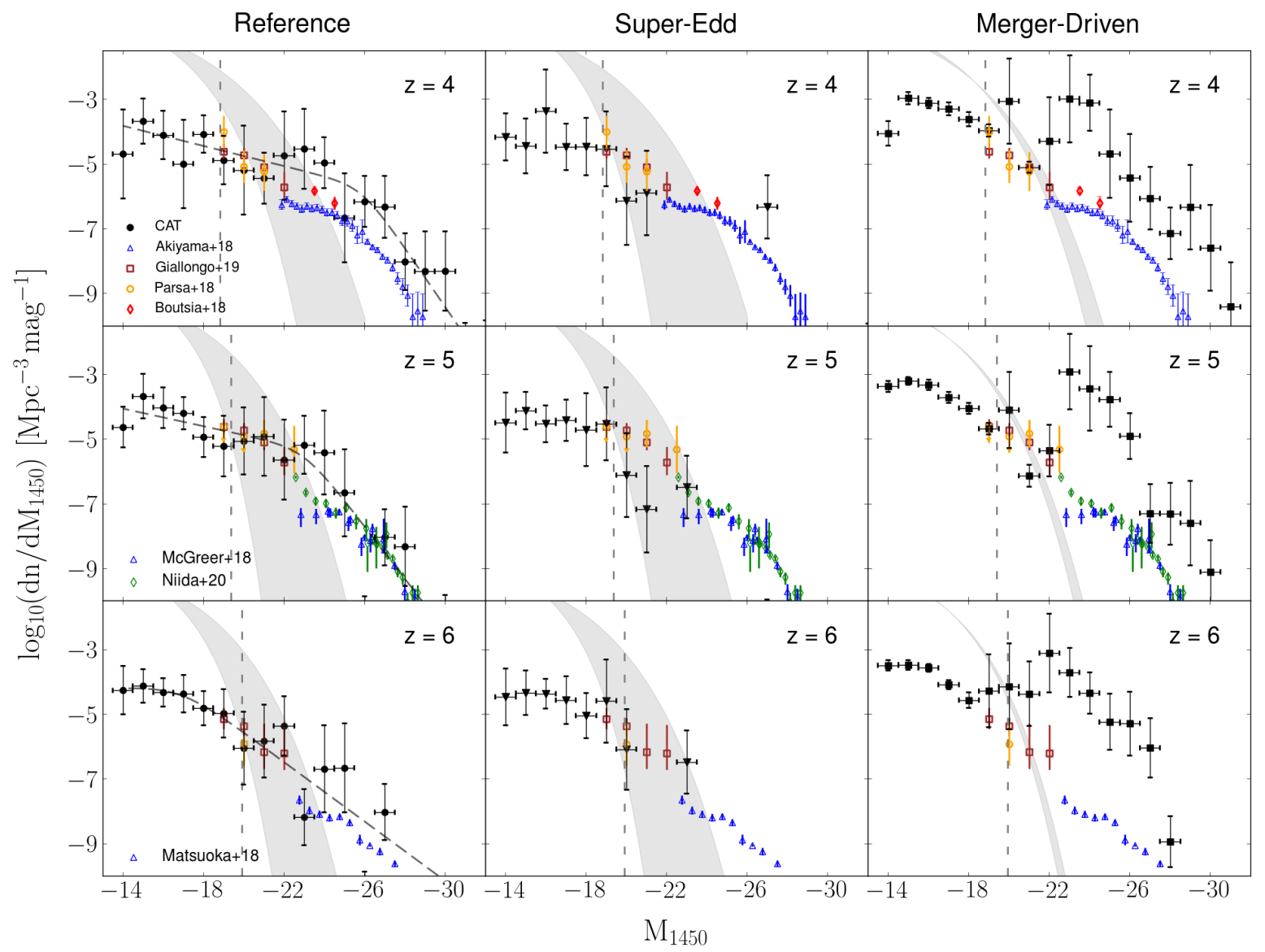

Figure 9. The AGN UV LFs at $z=4,5$, and 6 (from top to bottom). Each column shows the results obtained in one of the three cat models: the reference model (left panels), the super-Edd model (central panels) and the merger-driven model (right panels). Black data points represent the predicted AGN LFs, properly corrected for obscuration as described in the text. The black dashed line shows the best fit for the Reference model. The grey shaded area encloses the region between the intrinsic and the dust corrected galaxy UV luminosity function predicted by each model at the corresponding redshift. Coloured data represent the observational constraints on he AGN LF obtained by Parsa et al. (2018, orange circles), Boutsia et al. (2018, red diamonds), Giallongo et al. (2019, brown squares), by the SHELLQs survey (Matsuoka et al. 2018), the CFHT Legacy Survey (McGreer et al. 2018), and the Hyper Suprime-Cam Wide Survey (Akiyama et al. 2018; Niida et al. 2020) (blue triangles and green diamonds). The vertical black dashed lines show the JWST luminosity limit at each redshift predicted by Griffin et al. (2020).

contribution of the population of heavily-obscured Compton-thick AGNs, which might be relevant especially at lower luminosities. In Fig. 10, the luminosity limits reported by Griffin et al. (2020) for the Athena and Lynx observatories are also shown, at each redshift, with dashed and dotted grey vertical lines, respectively.

In the X-ray band, the reference model provides again a very close agreement with the empirical data. The predicted LF well matches the observations in the entire luminosity range explored, $\sim 10^{42}-10^{46} \mathrm{erg} \mathrm{s}^{-1}$, and predicts similar values for the break magnitude of the double power-law distribution at all redshifts.

The X-ray LF produced by the super-Edd model has a shape similar to the reference one, but systematically under-predicts the number of bright sources above $10^{43} \mathrm{erg} \mathrm{s}^{-1}$.

Finally, the merger-driven model predicts a peculiar luminosity distribution. Below $\sim 10^{44} \mathrm{erg} \mathrm{s}^{-1}$, the X-ray LF has a shape similar to the reference model but with a slightly larger amplitude. At higher luminosities the distribution is dominated instead by the large number of systems undergoing the bursty post-merger accretion phase, leading to a large scatter and to an over-estimation of the bright-end of the X-ray LF. However, we have to be careful in comparing with empirical data the luminosity of systems during such brief phases of merger-driven accretion. In fact, if these bursts of accretion take place on timescales much shorter than the typical time-step of our model, the time interval $t_{Q}$ (presented in Eq. 24) during which the $\mathrm{BH}$ undergoes an enhanced accretion will be overestimated, leading to an higher number of sources in the brightend of the AGN LF. In addition, these rapidly accreting systems are supposed to be highly affected by obscuration, which would furtherly shorten the duration of the observable burst of luminosity.

A gap in the X-ray LFs appears for both the reference and the Super-Edd models, just below a luminosity of $\sim 10^{41} \mathrm{erg} \mathrm{s}^{-1}$, as a consequence of the inefficient accretion of light black hole seeds. Starting from the same luminosity, we observe also in the mergerdriven variant a declining number of sources toward the faint-end 
of the distribution. In contrast with the previous case, this is due to the very efficient growth of light seed descendants coupled with the increasingly less probable seed formation at lower redshift, as we already observed in Fig. 4. Nevertheless, the merger-driven model shows a significantly smoother decline with respect to the marked gap observed in the reference scenario. These different predictions might potentially be used to discern between different accretion mechanisms with future surveys exploring such faint luminosities, as will be discussed in the next section.

\subsubsection{Predictions for future surveys}

Distinctive features in the AGN X-ray LFs, characterizing the underlying model of accretion, might represent a key element to discern between different possible scenarios for early BH growth. According to CAT model predictions, such differences should affect primarily the evolution of lower mass BHs, requiring observational constraints at very faint luminosities, $L_{\mathrm{X}} \lesssim 10^{41} \mathrm{erg} \mathrm{s}^{-1}$. It is interesting to note that, as shown in Fig. 10, sources with an X-ray luminosity of $\sim 10^{41} \mathrm{erg} \mathrm{s}^{-1}$ might still be observable with the next generation X-ray observatory Lynx, which will explore the faintend of the LFs, constraining the evolution of light black hole seeds and their dominant accretion mode. Athena observations will be restricted instead to brighter X-ray sources, constraining the distribution above $10^{43} \mathrm{erg} \mathrm{s}^{-1}$ (Griffin et al. 2020).

Still, it will be very challenging to investigate the AGN LFs sufficiently in detail to discern between different BH evolutionary scenarios. In fact, from Fig. 10 we observe that at luminosities $\leq 10^{41} \mathrm{erg} \mathrm{s}^{-1}$ the X-ray emission produced by stellar binaries formed in the host galaxies becomes comparable to the one contributed by AGNs. Therefore, the investigation of the X-ray luminosity distribution at such faint luminosities has to be carried out with methods that carefully take into account potential contamination from star formation in the host galaxies, in order to reliably discern between different $\mathrm{BH}$ growth modes. Moreover, the presence of a clear gap in the observed AGN number density below a given luminosity, as predicted in our reference model, might be covered, in future observations, by large scatters in the theoretical scaling relations or observational parameters assumed to estimate the empirical LFs.

In order to investigate the observational capability of the Athena and Lynx missions at even higher redshifts, in Fig. 11 we compare their forecast sensitivity in the redshift range $\mathrm{z} \in[6,7]$ and $[7,8]$ with the AGN X LFs predicted by the CAT reference and merger-driven models at $z \sim 7$ and 8 , accounting for obscuration. Similarly to the LFs shown at $z \leq 6$, a major difference between the two models is noticeable at the faint-end of the LFs, where the reference model shows a steeper decrease in the number density of fainter objects, due to the inefficient growth of light seeds descendants. This feature, however, appears just below the forecast sensitivity limit of Lynx and might be challenging to identify even with such deep observations. Despite that, our predictions suggest that a mission with sensitivity comparable to Lynx would have the potential to unveil a large population of AGNs, even at $z \geq 6-8$. This would open up the possibility to explore BHs with mass $\sim 10^{5}-10^{6} \mathrm{M}_{\odot}$, which dominate the AGN population at $z \leq 15$, regardless of the assumed $\mathrm{BH}$ accretion model.

At these high redshifts, the forecast sensitivity of Athena would enable to explore only the bright-end of the distribution, at $L_{\mathrm{X}}>10^{44} \mathrm{erg} \mathrm{s}^{-1}$. While this hampers the possibility of detecting the dominant $\mathrm{BH}$ population at $z \sim 7-8$, an interesting feature appears $L_{\mathrm{X}} \gtrsim 10^{43} \mathrm{erg} \mathrm{s}^{-1}$, where the merger-driven model predicts a much larger number density of sources compared to the reference one. This is a consequence of the growing rate of galaxy mergers with increasing redshift, which causes a larger fraction of AGNs to experience enhanced, super-Eddington accretion, increasing their luminosity. Such a distinctive feature could be potentially observable by Athena. Therefore, the observation of a slower decline of the AGN X-ray LF at higher redshifts might be a hint that early BH evolution is strongly driven by short period of enhanced accretion occurring during galaxy mergers. An important caveat here is that the predicted luminosity (and observability) of such rapidly accreting BHs might be affected by the short timescale of the process and by additional gas obscuration in the nuclear regions, as discussed in Section 4.2.2.

In the bottom panel of Fig. 11 we also show the percentage of BHs with at least one heavy seed progenitor in each luminosity bin. We observe that, in contrast with the results of the reference model, where all the AGNs are predicted to descend from at least one heavy $\mathrm{BH}$ seed, in the merger-driven scenario, more than $50 \%$ of AGNs descend from light BH seeds, even at the brightest X-ray luminosities. When $\mathrm{BH}$ growth can exceed the Eddington limit and it is triggered by galaxy mergers, the fraction of AGNs descending from heavy $\mathrm{BH}$ seeds is sub-dominant and decreases with redshift.

\subsubsection{Black hole - galaxy scaling relations}

In addition to the $\mathrm{BH}$ mass and luminosity distribution, we explored CAT predictions for the BH-galaxy scaling relations. In particular, it is interesting to understand if and how these relations are affected by the different $\mathrm{BH}$ growth scenario assumed in this work.

In Figure 12 we show the $M_{*}-M_{\mathrm{BH}}$ relation for our sample of galaxies at $z=5,6$ and 7. Left and right columns represent, respectively, the results obtained in the reference and merger-driven models. We compare CAT predictions with several empirical relations based on AGN and galaxy observations in the local Universe, investigating both the unobscured (Reines \& Volonteri 2015; Shankar et al. 2016; Greene et al. 2016; Suh et al. 2020) and obscured (Baron \& Ménard 2019) AGN population. We also assume as a reference the predictions obtained from the empirical model recently presented by Zhang et al. (2021) at the redshifts of interest.

The CAT reference model shows again a clear gap around $M_{\mathrm{BH}} \sim 10^{4} \mathrm{M}_{\odot}$, which splits the two populations of galaxies hosting a light or a heavy $\mathrm{BH}$ seed descendant. Note that, in less massive galaxies, below $M_{*} \lesssim 10^{9} \mathrm{M}_{\odot}$, the stellar mass is largely independent of the nature of the nuclear BH seed. The population of massive halos with $M_{*} \gtrsim 10^{9} \mathrm{M}_{\odot}$ hosts instead more massive BHs, showing a correlation between the two quantities. Remarkably, the galaxy population obtained by the CAT reference model closely reproduces the relations proposed by the empirically-constrained models assumed as comparison.

The merger-driven model shows instead a continuous relation between the mass of the galaxy and that of its nuclear BH with an increasing scatter for lower mass galaxies. It is interesting to note that, also in this model, the galaxy population lies on a slope which is very similar to the ones predicted by numerical models. The very early growth of BHs, which characterize this model variant, is clearly noticeable at $z=7$, where massive BHs populate less massive galaxies compared to the reference model. This is probably a natural consequence of the more competitive $\mathrm{BH}$ accretion model assumed, which impacts on the efficiency of star formation especially at early times. However, at lower redshift galaxies quickly increase their stellar mass, leading to a final distribution at $z \sim 5$ 


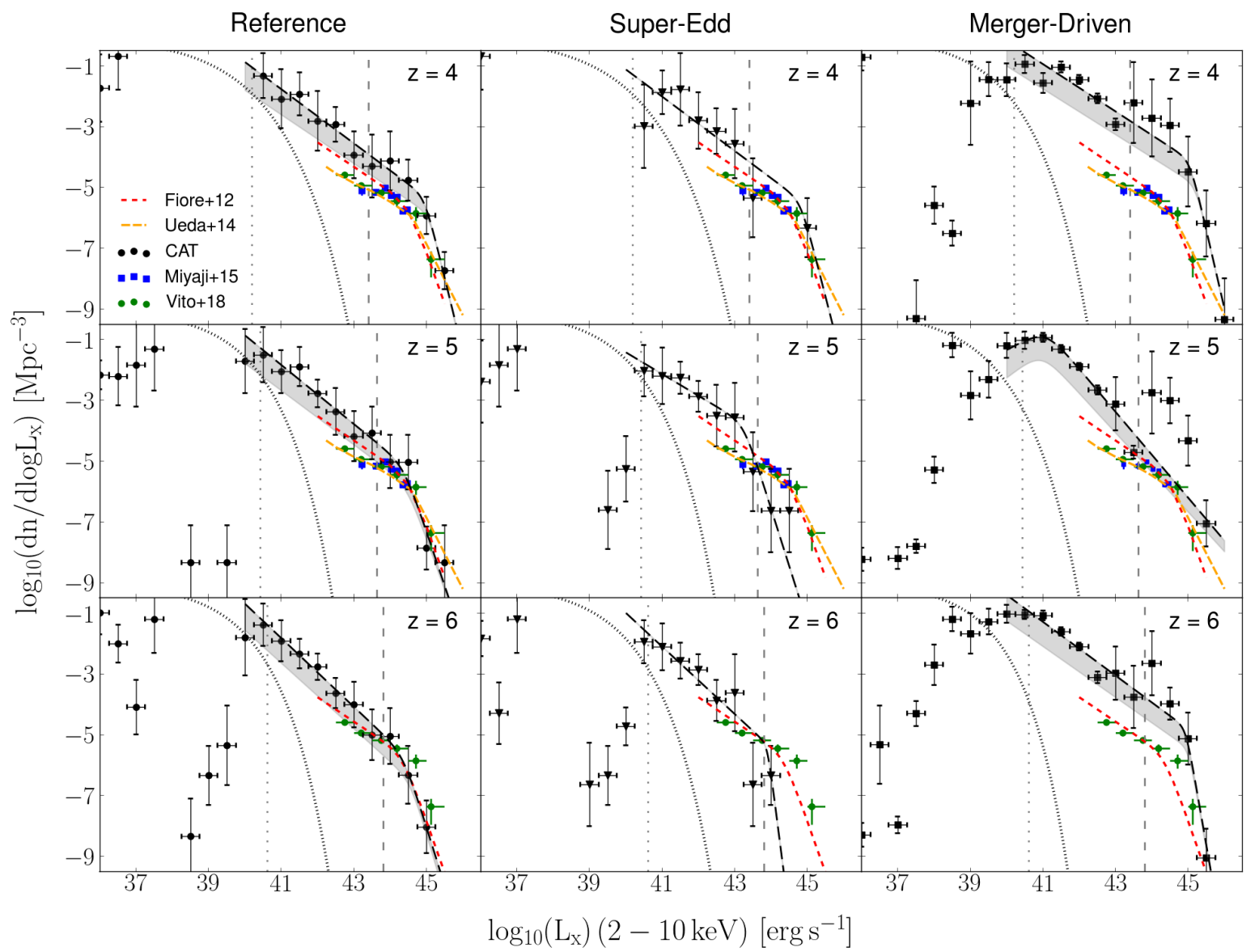

Figure 10. The AGN LFs in the [2 - 10] keV X-ray energy band at $z=4,5$, and 6 (from top to bottom). Each column shows the results obtained in one of the three models examined: the reference model (left panels), the super-Edd model (central panels) and the merger-driven model (right panels). In the left and right panels, the grey shaded regions show how the best-fit distributions change assuming the fraction of absorbed AGNs proposed by Ueda et al. (2014). In each panel, the black dotted line represents the X-ray LF associated with star formation in the host galaxies at the same redshift, that we have estimated using the relation proposed by Fornasini et al. (2018, see text). Coloured data represent the observational results by Fiore et al. (2012, red dashed line, $z=4.5,6$ ), Ueda et al. (2014, orange dashed line, $4<z<5$ ), Miyaji et al. (2015, blue squares, $3<z<5.8$ ) and Vito et al. (2018, green circles, $3.6<z<6$ ). The dashed and dotted grey vertical lines represent the luminosity limits estimated by Griffin et al. (2020) for, respectively, Athena and Lynx.

which presents an offset of $\lesssim 1$ dex compared to the prediction of empirical models.

The above results show that even very different paradigms for $\mathrm{BH}$ growth, as the ones considered in the reference and mergerdriven models, lead to similar properties for the galaxy and $\mathrm{BH}$ populations at $z \sim 4-5$. Deep sky observations at higher redshift will be hence crucial to better understand the nature of the first $\mathrm{BH}$ seeds and their co-evolution with the host galaxy.

\section{DISCUSSION}

In this section, we first compare the results of CAT with independent numerical and semi-analytical studies. We then discuss the main caveats of the model and how we plan to address these in the future.

\subsection{Comparison with previous studies}

The formation and evolution mechanisms of supermassive black holes at high redshift have been the focus of several studies in the last few years. Despite very challenging, observational constraints on the BH mass function at redshift $z \gtrsim 4$ have been proposed by different works, often relying on the correlations between the black hole mass and the properties of the SMBH host (Shankar et al. 2009, 2010; Merloni \& Heinz 2008; Willott et al. 2010b). However, the scaling relations between the $\mathrm{BH}$ mass and the host galaxy are mainly determined in the local universe, while their evolution in redshift is still largely uncertain. Hence, in order to reconstruct the $\mathrm{BH}$ mass function at higher redshift, these works usually rely on the AGN luminosity distribution as a tracer of the accretion history of SMBHs, assuming the local BH distribution as a boundary condition. Unfortunately, this requires some assumptions on the efficiency of the $\mathrm{BH}$ accretion process, such as duty cycle, radiative efficiency, obscured AGN fraction, etc., leading to discrepancies 


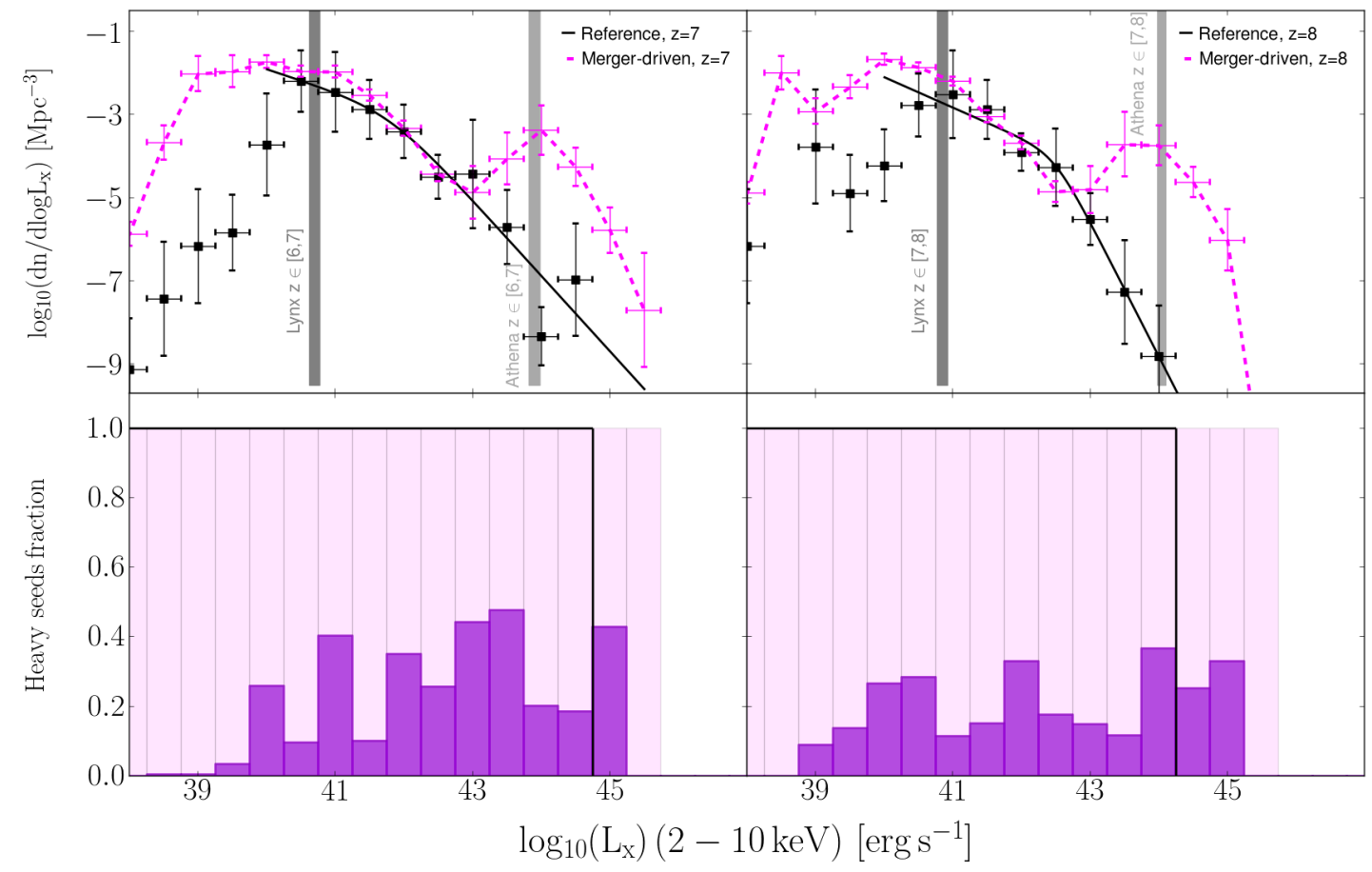

Figure 11. Top panels: AGN X-ray LFs predicted by CAT at $z \sim 7,8$. The black data points are the results of our reference model, with the black solid curves representing the best fit of the bright-end distribution at each redshift. As a comparison, we also show the results of the merger-driven model with dashed magenta lines and error bars. The sensitivity limits proposed by (Griffin et al. 2020) for a mission like Lynx and for Athena in the redshift ranges $\mathrm{z} \in[6,7],[7,8]$ are shown, respectively, as dark grey and light grey vertical thick lines. Bottom panels: percentage of heavy BH seed descendants in each luminosity bin, for the reference (black lines) and merger-driven (violet histograms) models. In the reference model, all the $\mathrm{AGNs}$ with $\log _{10}\left(\mathrm{~L}_{\mathrm{X}}\right)>38$ have at least one heavy seed progenitor.

between different results (see Kelly \& Merloni 2012, for a detailed discussion). This point has to be carefully taken into account in the comparison between the observational constraints and the intrinsic mass function obtained by theoretical models as cAT. Important efforts on the SMBHs evolution have been carried out also through large-scale cosmological simulations. This approach starts usually with a given cosmological framework and follows the baryonic evolution by zooming in particularly dense regions where the massive nuclear black holes are supposed to form. This class of simulations has shown to be able to characterize properly the evolution of a wide range of massive black holes. However, it still hardly succeeds in reproducing the most extreme sources that we observe at high redshift, which reach masses above $10^{9} \mathrm{M}_{\odot}$ already at $z \geq 7$ (Mortlock et al. 2011; Yang et al. 2020; Wang et al. 2021). This is probably due to the large volume needed to resolve the rare overdense regions where the assembly of the most massive SMBHs can take place Tenneti et al. (see e.g. 2018, 2019). The limited volume of this class of simulations leads indeed to a significant underestimation of the number of rare and bright AGNs at very high redshifts, which consequentially affects the predicted mass and luminosity functions (Amarantidis et al. 2019).

In Fig. 13 we compare the binned mass function at redshift $z=4$ obtained by CAT reference model with the results of some of the most recent and important large-scale cosmological simulations, namely the Illustris (Sijacki et al. 2015), IllustrisTNG (Weinberger et al. 2017), Horizon-AGN (Volonteri et al. 2016), SIMBA (Davé et al. 2019; Thomas et al. 2019) and EAGLE (McAlpine et al. 2017, 2018). For completeness, we also show the observational constraints obtained from Merloni \& Heinz (2008) and Shankar et al. (2009). We can see that the MF predicted by numerical simulations covers a smaller range of $\mathrm{BH}$ masses with respect to our semi-analytical approach. While at the high-mass end of the distribution this is due to the limited simulation volume, below $\sim 10^{6} \mathrm{M}_{\odot}$ the nuclear $\mathrm{BH}$ population can not be properly modelled due to the resolution limits of the simulations, which dictate the seeding prescription. In fact, since large-scale simulations are not able to well resolve lower mass galaxies, they are forced to seed more massive galaxies with $M_{*} \gtrsim 10^{9} \mathrm{M}_{\odot}$ with nuclear BHs with mass $\sim 10^{5-6} \mathrm{M}_{\odot}$ (Habouzit et al. 2020).

The figure shows that numerical simulations provide consistent results at the high mass end of the distribution, above $10^{8} \mathrm{M}_{\odot}$, while they differ mostly at lower masses, probably as a result of the different sub-grid physics and seeding prescriptions adopted. The BH mass function obtained in our reference model is in broad agreement with the results of the numerical simulations, especially in the $\mathrm{BH}$ mass range $\left[10^{6}-10^{8}\right] M_{\odot}$, where it is consistent with the observationally constrained MF of Merloni \& Heinz (2008). At higher mass values, where the contribution of the most massive and rarer systems becomes relevant, both САT and numerical simulations seem instead to slightly overestimate the number of SMBHs with respect to Merloni \& Heinz (2008). Fitting our binned SMBH mass function with a power law, though, a better agreement is found with 


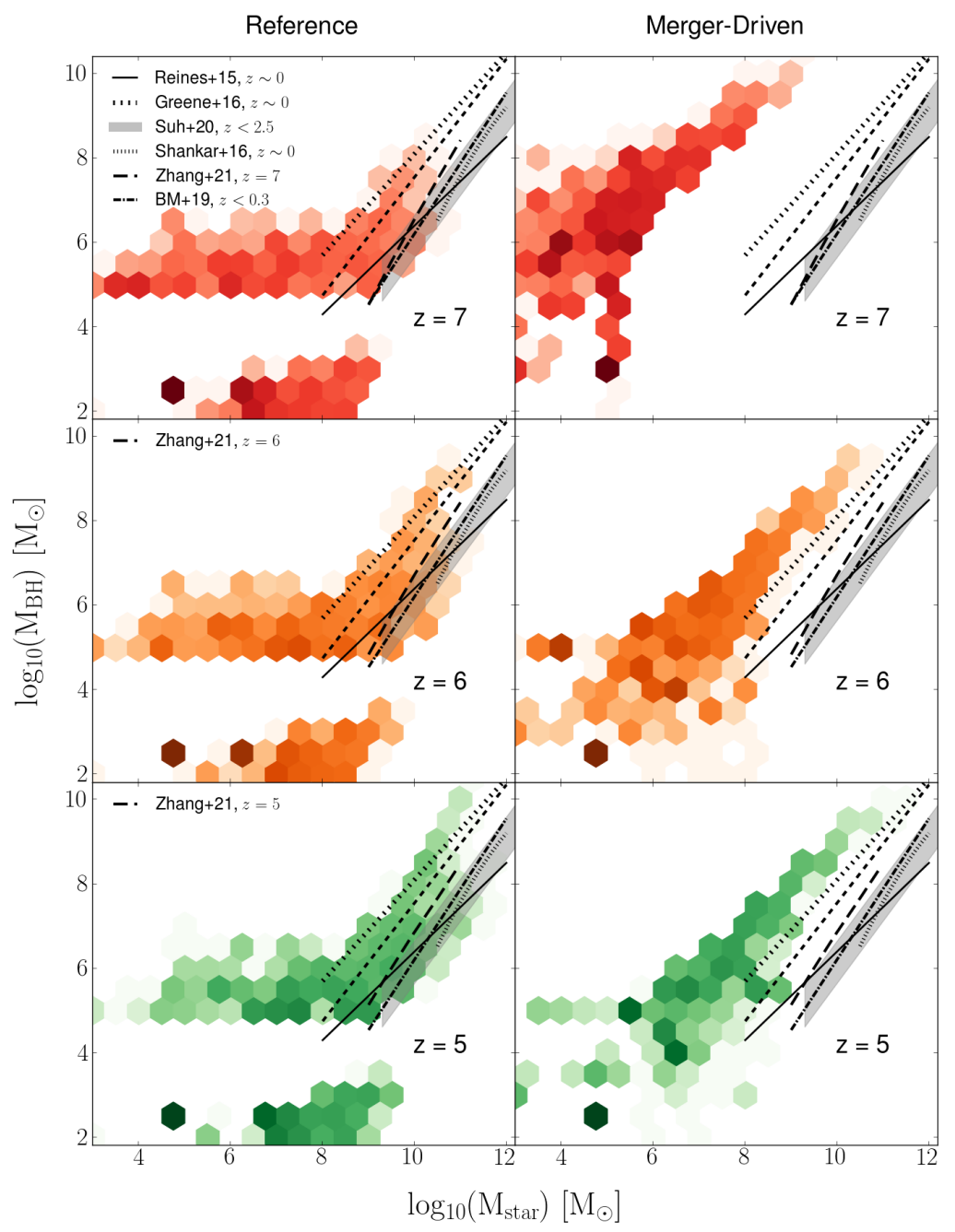

Figure 12. Black hole mass as a function of the galaxy stellar mass for the cAT galaxy population at different redshifts, $z=5,6,7$. Left and right panels represent, respectively, the results obtained in the reference and merger-driven models. CAT results are compared with the predictions of the empirical models proposed by Reines \& Volonteri (2015, solid and dashed lines for, respectively, AGNs and elliptical galaxies), Shankar et al. (2016, dotted line), Greene et al. (2016, loosely dotted line), Baron \& Ménard (2019, dash-dotted line), Suh et al. (2020, grey shaded area) and Zhang et al. (2021, long dashed line).

the observational constraints obtained by Shankar et al. (2009) in the high-mass range, above $\sim 10^{9} \mathrm{M}_{\odot}$.

In Fig.13, for comparison, we also show the best fit of the BH mass function obtained by CAT merger-driven model. As anticipated in the previous sections, this model variant predicts a significantly larger number density of BHs with masses $\lesssim 10^{9} M_{\odot}$ compared to the reference model and to the results of numerical simulations and observational studies. This is probably a consequence of the importance that light seeds acquire in this alternative scenario, where they are able to efficiently grow across cosmic time and contribute in the building up of the entire $\mathrm{BH}$ mass function. It has to be pointed out, however, that the mass function predicted by our merger-driven scenario at $z \sim 4$ seems in tension with the estimate local BH mass density inferred from the $M_{\mathrm{BH}}-M_{*}$ relation (see e.g. the recent work of Shankar et al. 2020). This suggests that a more refined modeling of the black hole accretion process, as the one originally proposed by Pezzulli et al. (2016), which assumes a distinguished treatment for the galaxy bulge and disk environments, would be required in this scenario. That, in fact, might ensure a better consistency with the AGN mass and luminosity distributions, maintaining at the same time a good accordance with the global constraints.

In the bottom panel of Fig.13, we also compare our results at $z=5$ with similar semi-analytical studies. We show in particular the results of different SMBH evolutionary models studied by Ricarte \& Natarajan (2018a), considering both burst and steady mode accretion. Despite their focus is on massive BH seeds, mainly DCBHs or extremely fast-growing Pop III remnants, their results in the mass range $\left[10^{7}-10^{10}\right] M_{\odot}$ are in very good agreement with CAT predictions. Interestingly, their results fall just between the best-fit of our reference and merger-driven models, as we would expect for a mixed accretion scenario as the one considered there.

CAT predictions are also compared with the results of the Del- 


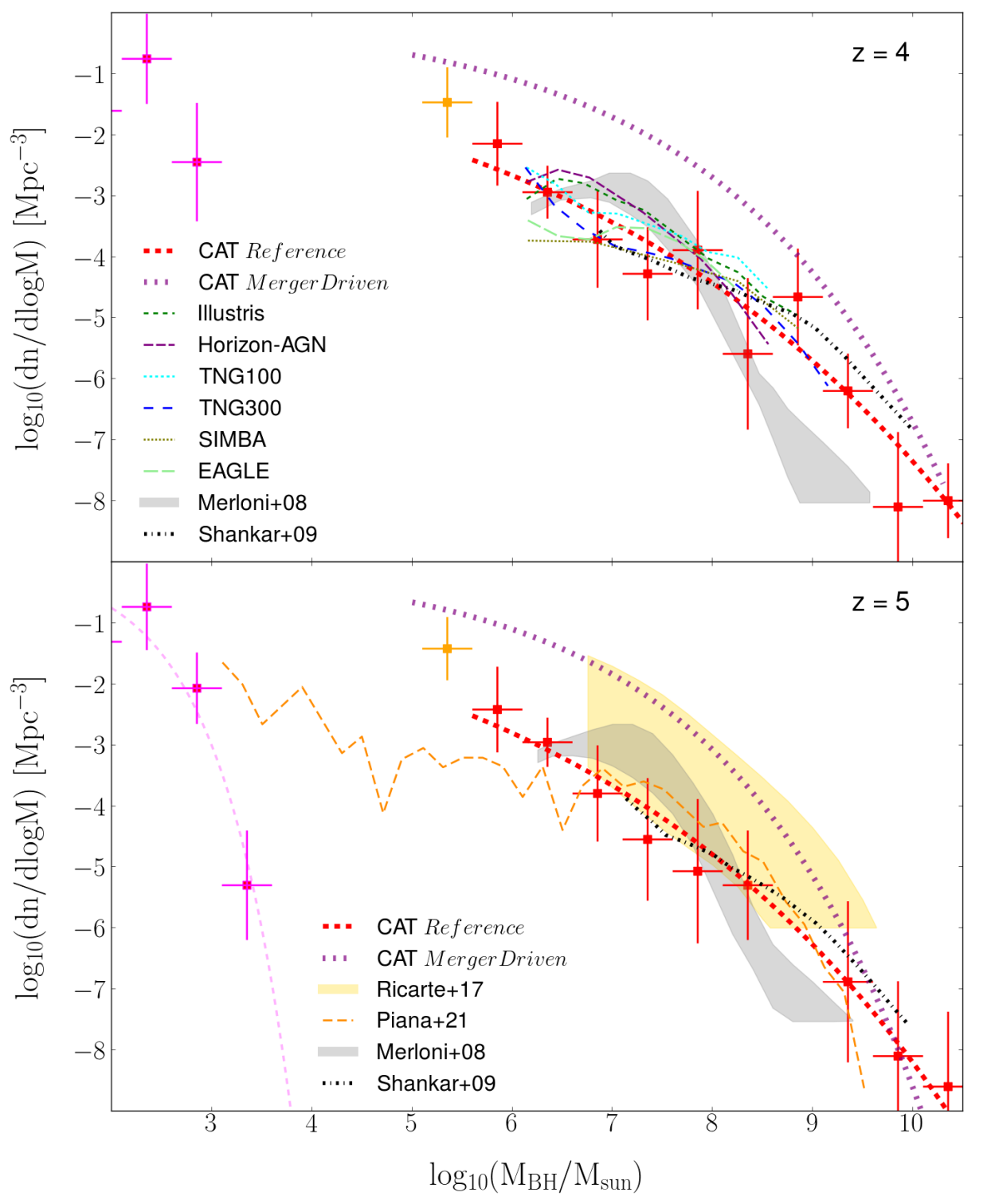

Figure 13. Comparison between the mass function predicted by CAT and the results of numerical simulations (upper panel, $z=4$ ) and semi-analytical models (lower panel, $z=5$ ). As in Fig.4, the data points show the binned distribution obtained by CAT reference model for light (magenta) and heavy (red, orange) BH descendants, with the red dashed line representing the best-fit of the distribution of heavy seed descendants. The violet dotted lines show the best-fit of the BH MF obtained by the merger-driven model. In the upper panel, as a comparison, we present with colored dashed lines the results of large-scale cosmological simulations, namely: Illustris (dark green), IllustrisTNG100 (cyan), IllustrisTNG300 (blue), SIMBA (olive) and EAGLE (light green). In the lower panel we show the mass function obtained by the semi-analytical models by Ricarte \& Natarajan (2018a, yellow shaded region) and Piana et al. (2021, orange dashed line). The grey shaded area and the dashed-dotted black lines represent instead the observational constraints proposed by Merloni \& Heinz (2008) and Shankar et al. (2009) respectively.

phi semi-analytic model proposed by Piana et al. (2021). The two models implement similar approaches in investigating the BH mass distribution, following the evolution of both stellar and direct collapse $\mathrm{BH}$ progenitors in a cosmological framework. However, CAT and Delphi present considerably different prescriptions for $\mathrm{BH}$ seed formation and growth. In fact, in the latter, BH seeding is less sen- sitive to the galaxy environmental conditions, since all dark matter halos are initially seeded with a stellar $\left(150 \mathrm{M}_{\odot}\right)$ or a DCBH seed $\left(10^{3-4} \mathrm{M}_{\odot}\right)$, depending only on the initial incident $\mathrm{LW}$ radiation. The newly formed BHs are then assumed to accrete a given fraction of the available gas, independently of their mass, without exceeding the Eddington limit. Despite these differences, we find consistent 
results for the $\mathrm{BH} \mathrm{MF}$ at $z \sim 5$ in the whole range of masses above $\sim 10^{6.5} M_{\odot}$. Interestingly, they do not find any gap in the mass function at lower BH masses. This is probably due to the different $\mathrm{BH}$ accretion mode adopted in Delphi, which does not depend on the actual $\mathrm{BH}$ mass but only on the available gas mass in the host halo, at odds with our reference and super-Edd models, where $\mathrm{BHs}$ are assumed to grow at the Bondi-Hoyle rate. In addition, in Delphi the initial mass assumed for heavy (direct collapse) BHs is different from what we adopt in CAT: while we consider an initial mass of $10^{5} \mathrm{M}_{\odot}$, in the center of the supposed mass range for this class of seeds (Latif et al. 2016; Becerra et al. 2018), Piana et al. (2021) rely on a more conservative value of $10^{3-4} \mathrm{M}_{\odot}$. This difference translates into a continuous mass function ranging between $\sim 10^{3}-10^{10} \mathrm{M}_{\odot}$ and possibly accounts for the observed flattening in the mass function with respect to CAT predictions at $M_{\mathrm{BH}} \lesssim 10^{6.5} \mathrm{M}_{\odot}$. This comparison suggests that the initial mass of heavy BH seeds has a strong influence on the shape of the AGN mass and luminosity distributions. In particular, a smaller birth mass of heavy seeds might reduce the predicted gap in the MF and, therefore, have an impact on the observability of the distinctive features characterizing different accretion models. However, it is important to note that predicting the initial mass function of heavy BH seeds is very challenging as this likely depends on the adopted conditions for their formation (Ferrara et al. 2014; Bhowmick et al. 2021).

\subsection{Main caveats of the model}

Despite the successes of CAT model predictions in reproducing a wide range of observational constraints (see Section 3) and the good agreement with independent studies discussed above, we plan to overcome some of the limitations that we have already anticipated in the previous sections and that we discuss below. The first one is the adopted seeding prescription. Although our model allows us to describe the formation of light and heavy $\mathrm{BH}$ seeds depending on the properties of their birth environment, we plan to expand our model following the approach of Sassano et al. (2021) to include the formation of medium-weight $\mathrm{BH}$ seeds by runaway stellar collisions in dense star clusters. This will allow us to track the formation and mass growth of three independent families of $\mathrm{BH}$ seeds in a full cosmological context and to predict their observational signatures.

The paradigm assumed for the $\mathrm{BH}$ accretion in low mass galaxies represents an additional crucial point. As observed in Section 4.2, the LFs obtained in the CAT reference model predicts an excess of faint sources, below $\sim 10^{42} \mathrm{erg} \mathrm{s}^{-1}$, if compared to observations. Despite empirical data are not strongly constrained at such low luminosities, this might be a consequence of considering an accretion model which is too efficient for BHs growing in low mass galaxies. In similar semi-analytic models that are calibrated to reproduce the AGN LF at lower redshift, e.g. Piana et al. (2021), BH accretion is in fact artificially inhibited below a typical halo mass of $\sim 10^{12} \mathrm{M}_{\odot}$ to mimic the effect of SN feedback suggested by numerical studies (Habouzit et al. 2017; Anglés-Alcázar et al. 2017). As shown in Figure 13 this lead to a lower number density of BHs below $10^{7} \mathrm{M}_{\odot}$ compared to CAT predictions. In future works, an improved prescription for $\mathrm{BH}$ accretion, assuming in particular an $\alpha_{B H}$ parameter (Eq. 12) which depends on the properties of the host galaxies, as proposed in Booth \& Schaye (2009), will be crucial to better understand the nature of these discrepancies.

A further improvement of the model will be to include the effects of BH dynamics during galaxy mergers. As discussed in Section 2.3.3, in the current version of CAT, two BHs are assumed to merge only during major mergers of their dark matter host halos, while in minor mergers the heaviest $\mathrm{BH}$ is assumed to migrate to the center of the newly formed galaxy while the lightest one remains a satellite and we do not follow further its evolution. A more physical description of $\mathrm{BH}$ dynamics requires to take into account the impact of processes dominating on different spatial scales/cosmic epochs (Begelman et al. 1980; Armitage \& Natarajan 2002, 2005; Colpi 2014): dynamical friction against background gas and stars regulate the sinking timescale of BHs on kpc-to-pc scales, determining whether a bound system can form (e.g. Capelo et al. 2015; Tamburello et al. 2017; Pfister et al. 2017, 2019; Tamfal et al. 2018; Bortolas et al. 2020; Barausse et al. 2020, and references therein). On smaller scales, interactions with gaseous disks, stars and other $\mathrm{BHs}$ (triple/multiple interactions) instead control the duration of the binary BH hardening phase (e.g. Bortolas et al. 2016, 2018; Biava et al. 2019; Arca Sedda et al. 2019; Souza Lima et al. 2020, and references therein). We plan to study these aspects in future works, building on the first implementation of $\mathrm{BH}$ dynamics (triple $\mathrm{BH}$ interactions) in GQD recently proposed by Valiante et al. (2020).

\section{SUMMARY AND CONCLUSIONS}

In this work we have used the Cosmic Archaeology Tool semianalytical model to explore how different $\mathrm{BH}$ accretion and feedback prescriptions affect the formation and evolution of the first galaxies and their nuclear BHs from $z \sim 25$ to $z \sim 4$. In particular, our aim was to use CAT has a laboratory to test whether the nature of the first $\mathrm{BH}$ seeds and their growth mode may leave observable imprints on the $\mathrm{BH}$ mass and luminosity functions. Note that, unlike the majority of numerical and semi-analytic models presented and used as a comparison in Section 5.1, CAT follows the formation of both light and heavy $\mathrm{BH}$ seeds, with a seeding prescription that depends on the physical conditions at their formation sites. This enables us to investigate how these two seed populations contribute to the statistical properties of the BH population at $4 \leq z \leq 6$.

We explored both a classic BHL accretion scenario, assumed as our reference model, and two model variants: the super-Edd model, where we removed the Eddington limit for $\mathrm{BH}$ accretion, and the merger-driven model, where - in addition - enhanced $\mathrm{BH}$ accretion episodes are triggered by galaxy mergers.

The most important results of this work are summarized below.

- The reference model shows the best agreement with observational data. The predicted $\mathrm{BH}$ luminosity function is in good agreement with several empirical constraints at $z=4,5,6$, especially at higher luminosities. At the faint-end, close to the current observational limits, CAT slightly over-predicts the number of sources, suggesting an overstated growth for less massive BHs. The predicted $\mathrm{BH}$ mass function is consistent with independent numerical models, as well as with different empirical constraints, although the agreement is limited to specific mass ranges as these empirical constraints do not always provide consistent results.

- The super-Edd model seems instead to fail at reproducing the observations. The lack of an Eddington-limit leads to an accelerated early growth of BH seeds and thus to a quick depletion of gas inside their host galaxies. These conditions strongly affect the subsequent growth of nuclear BHs, which fail to reach both masses and luminosities of the most extreme quasars observed at $z \gtrsim 6$.

- The merger-driven model predicts global trends for the mass and luminosity distributions very similar to the results of the 
reference model, despite not entirely consistent with empirical constraints and observational data. This tension might be also due to an intrinsic limit, since we implemented a simplified version of the original model developed by Pezzulli et al. (2016). In future works, refined prescriptions for $\mathrm{BH}$ accretion, as well as a more accurate treatment of the involved timescales, will enable us to derive tighter constraints.

- We find that the main difference between the reference and merger-driven accretion models lies in the evolution of the light $\mathrm{BH}$ seed population. In the first model, their stunted growth leads to a clear gap in the resulting mass and luminosity functions, while in the second model they are able to grow in gas-rich galaxy mergers resulting into continuous $\mathrm{BH}$ mass and luminosity distributions where both light and heavy $\mathrm{BH}$ seeds can contribute to the same mass and luminosity bins.

- The signature of the BH seeds growth mode is imprinted in the BH luminosity function at the very faint end, in a luminosity regime that will be extremely challenging to test observationally. In the X-ray, a mission with a sensitivity comparable to the Lynx $\mathrm{X}$-ray observatory might be able to probe the $z \sim 4-6$ luminosity function at $L_{\mathrm{X}} \leq 10^{41} \mathrm{erg} / \mathrm{s}$, possibly unveiling precious hints on the $\mathrm{BH}$ accretion mechanism. Interestingly, we find that at $z \geq 6-8$ the forecast sensitivity of Athena could be enough to disentangle the signature of super-Eddington, merger-driven $\mathrm{BH}$ growth at $L_{X}>10^{43} \mathrm{erg} / \mathrm{s}$ by detecting a larger number of AGNs or a milder evolution at the bright-end of the X-ray LF compared to the predictions of the reference model.

\section{ACKNOWLEDGEMENTS}

We wish to thank the anonymous Referee for the constructive suggestions and insightful comments that improved the quality of the paper. We acknowledge support from the Amaldi Research Center funded by the MIUR program "Dipartimento di Eccellenza"(CUP:B81I18001170001), from the INFN TEONGRAV specific initiative, and the networking support by the COST Action CA16104. LZ acknowledges financial support under ASI/INAF contract 2017-14-H.0.

\section{DATA AVAILABILITY}

The simulated data underlying this article will be shared on reasonable request to the corresponding author.

\section{REFERENCES}

Abramowicz M. A., Czerny B., Lasota J. P., Szuszkiewicz E., 1988, ApJ, 332,646

Agarwal B., Khochfar S., 2015, MNRAS, 446, 160

Akiyama M., et al., 2018, PASJ, 70, S34

Amarantidis S., et al., 2019, MNRAS, 485, 2694

Ananna T. T., et al., 2019, ApJ, 871, 240

Andika I. T., et al., 2020, ApJ, 903, 34

Anglés-Alcázar D., Faucher-Giguère C.-A., Quataert E., Hopkins P. F., Feldmann R., Torrey P., Wetzel A., Kereš D., 2017, MNRAS, 472, L109

Arca Sedda M., Berczik P., Capuzzo-Dolcetta R., Fragione G., Sobolenko

M., Spurzem R., 2019, MNRAS, 484, 520

Armitage P. J., Natarajan P., 2002, ApJ, 567, L9
Armitage P. J., Natarajan P., 2005, ApJ, 634, 921

Bañados E., et al., 2016, ApJS, 227, 11

Bañados E., et al., 2018, Nature, 553, 473

Barausse E., Dvorkin I., Tremmel M., Volonteri M., Bonetti M., 2020, arXiv e-prints, p. arXiv:2006.03065

Baron D., Ménard B., 2019, MNRAS, 487, 3404

Baugh C. M., 2006, Reports on Progress in Physics, 69, 3101

Becerra F., Greif T. H., Springel V., Hernquist L. E., 2015, MNRAS, 446, 2380

Becerra F., Marinacci F., Bromm V., Hernquist L. E., 2018, MNRAS, 480, 5029

Begelman M. C., Blandford R. D., Rees M. J., 1980, Nature, 287, 307

Bellovary J., Volonteri M., Governato F., Shen S., Quinn T., Wadsley J., 2011, ApJ, 742, 13

Bhowmick A. K., Blecha L., Torrey P., Kelley L. Z., Vogelsberger M., Nelson D., Weinberger R., Hernquist L., 2021, arXiv e-prints, p. arXiv:2107.06899

Biava N., Colpi M., Capelo P. R., Bonetti M., Volonteri M., Tamfal T., Mayer L., Sesana A., 2019, MNRAS, 487, 4985

Boco L., Lapi A., Danese L., 2020, ApJ, 891, 94

Bondi H., 1952, MNRAS, 112, 195

Bonoli S., Mayer L., Callegari S., 2014, MNRAS, 437, 1576

Booth C. M., Schaye J., 2009, MNRAS, 398, 53

Bortolas E., Gualandris A., Dotti M., Spera M., Mapelli M., 2016, MNRAS, 461,1023

Bortolas E., Mapelli M., Spera M., 2018, MNRAS, 474, 1054

Bortolas E., Capelo P. R., Zana T., Mayer L., Bonetti M., Dotti M., Davies M. B., Madau P., 2020, arXiv e-prints, p. arXiv:2005.02409

Boutsia K., Grazian A., Giallongo E., Fiore F., Civano F., 2018, ApJ, 869, 20

Bouwens R. J., et al., 2012, ApJ, 754, 83

Bouwens R. J., et al., 2014, ApJ, 795, 126

Bromm V., 2013, Reports on Progress in Physics, 76, 112901

Bromm V., Loeb A., 2003, The Astrophysical Journal, 596, 34

Callegari S., Mayer L., Kazantzidis S., Colpi M., Governato F., Quinn T., Wadsley J., 2009, ApJ, 696, L89

Campanelli M., Lousto C., Zlochower Y., Merritt D., 2007, ApJ, 659, L5

Capelo P. R., Volonteri M., Dotti M., Bellovary J. M., Mayer L., Governato F., 2015, MNRAS, 447, 2123

Chehade B., et al., 2018, MNRAS, 478, 1649

Chon S., Omukai K., 2020, Supermassive Star Formation via Super Competitive Accretion in Slightly Metal-enriched Clouds (arXiv: 2001.06491)

Chon S., Hirano S., Hosokawa T., Yoshida N., 2016, ApJ, 832, 134

Chon S., Hosokawa T., Omukai K., 2021, MNRAS, 502, 700

Cole S., Lacey C. G., Baugh C. M., Frenk C. S., 2000, MNRAS, 319, 168

Cole S., Helly J., Frenk C. S., Parkinson H., 2008, MNRAS, 383, 546

Colpi M., 2014, Space Sci. Rev., 183, 189

Davé R., Anglés-Alcázar D., Narayanan D., Li Q., Rafieferantsoa M. H., Appleby S., 2019, MNRAS, 486, 2827

Dayal P., Ward M., Cockell C., 2016, arXiv e-prints, p. arXiv:1606.09224

Dayal P., Rossi E. M., Shiralilou B., Piana O., Choudhury T. R., Volonteri M., 2019, MNRAS, 486, 2336

Dayal P., et al., 2020, MNRAS, 495, 3065

De Lucia G., 2019, Galaxies, 7, 56

De Rosa G., Decarli R., Walter F., Fan X., Jiang L., Kurk J., Pasquali A., Rix H. W., 2011, ApJ, 739, 56

DeGraf C., Di Matteo T., Khandai N., Croft R., Lopez J., Springel V., 2012, MNRAS, 424, 1892

DeGraf C., Di Matteo T., Treu T., Feng Y., Woo J. H., Park D., 2015, MNRAS, 454, 913

Devecchi B., Volonteri M., 2009, The Astrophysical Journal, 694, 302

Devecchi B., Volonteri M., Rossi E. M., Colpi M., Portegies Zwart S., 2012, MNRAS, 421, 1465

Di Matteo T., Springel V., Hernquist L., 2005, Nature, 433, 604

Di Matteo T., Khandai N., DeGraf C., Feng Y., Croft R. A. C., Lopez J., Springel V., 2012, ApJ, 745, L29 
Di Matteo T., Croft R. A. C., Feng Y., Waters D., Wilkins S., 2017, MNRAS, 467,4243

Duncan K., et al., 2014, MNRAS, 444, 2960

Dunn G., Holley-Bockelmann K., Bellovary J., 2020, ApJ, 896, 72

Duras F., et al., 2020, A\&A, 636, A73

Eilers A.-C., Davies F. B., Hennawi J. F., 2018, ApJ, 864, 53

Eilers A.-C., et al., 2020, ApJ, 900, 37

Ellis R. S., et al., 2013, ApJ, 763, L7

Fan X., Narayanan V. K., Strauss M. A., White R. L., Becker R. H., Pentericci L., Rix H.-W., 2002, AJ, 123, 1247

Fan X., et al., 2003, AJ, 125, 1649

Feng Y., Di-Matteo T., Croft R. A., Bird S., Battaglia N., Wilkins S., 2016, MNRAS, 455, 2778

Ferrara A., Salvadori S., Yue B., Schleicher D., 2014, MNRAS, 443, 2410

Fiore F., et al., 2012, A\&A, 537, A16

Fornasini F. M., Civano F., Fabbiano G., Elvis M., Marchesi S., Miyaji T., Zezas A., 2018, ApJ, 865, 43

Giallongo E., et al., 2019, ApJ, 884, 19

González V., Labbé I., Bouwens R. J., Illingworth G., Franx M., Kriek M., 2011, ApJ, 735, L34

Grazian A., et al., 2015, A\&A, 575, A96

Graziani L., Schneider R., Ginolfi M., Hunt L. K., Maio U., Glatzle M., Ciardi B., 2020, MNRAS, 494, 1071

Greene J. E., et al., 2016, ApJ, 826, L32

Greif T. H., Springel V., White S. D. M., Glover S. C. O., Clark P. C., Smith R. J., Klessen R. S., Bromm V., 2011, ApJ, 737, 75

Griffin A. J., Lacey C. G., Gonzalez-Perez V., Lagos C. d. P., Baugh C. M., Fanidakis N., 2019, MNRAS, 487, 198

Griffin A. J., Lacey C. G., Gonzalez-Perez V., Lagos C. d. P., Baugh C. M., Fanidakis N., 2020, MNRAS, 492, 2535

Habouzit M., Volonteri M., Dubois Y., 2017, MNRAS, 468, 3935

Habouzit M., et al., 2019, MNRAS, 484, 4413

Habouzit M., et al., 2020, arXiv e-prints, p. arXiv:2006.10094

Haemmerlé L., Meynet G., Mayer L., Klessen R. S., Woods T. E., Heger A., 2019, A\&A, 632, L2

Haiman Z., Thoul A. A., Loeb A., 1996, ApJ, 464, 523

Heger A., Woosley S. E., 2002, ApJ, 567, 532

Hirano S., Hosokawa T., Yoshida N., Umeda H., Omukai K., Chiaki G., Yorke H. W., 2014, The Astrophysical Journal, 781, 60

Hirano S., Zhu N., Yoshida N., Spergel D., Yorke H. W., 2015, ApJ, 814, 18

Hirschmann M., Dolag K., Saro A., Bachmann L., Borgani S., Burkert A., 2014, MNRAS, 442, 2304

Hopkins P. F., Richards G. T., Hernquist L., 2007, ApJ, 654, 731

Hosokawa T., Omukai K., Yorke H. W., 2012, ApJ, 756, 93

Hosokawa T., Hirano S., Kuiper R., Yorke H. W., Omukai K., Yoshida N., 2016, ApJ, 824, 119

Hoyle F., Lyttleton R. A., 1941, MNRAS, 101, 227

Huang K.-W., Ni Y., Feng Y., Di Matteo T., 2020, MNRAS, 496, 1

Inayoshi K., Tanaka T. L., 2015, Monthly Notices of the Royal Astronomical Society, 450, 4350

Inayoshi K., Omukai K., Tasker E., 2014, MNRAS, 445, L109

Inayoshi K., Haiman Z., Ostriker J. P., 2016, MNRAS, 459, 3738

Inayoshi K., Visbal E., Haiman Z., 2019, arXiv e-prints, p. arXiv:1911.05791

Inayoshi K., Visbal E., Haiman Z., 2020, ARA\&A, 58, 27

Johnson J. L., Haardt F., 2016, Publ. Astron. Soc. Australia, 33, e007

Katz H., Sijacki D., Haehnelt M. G., 2015, MNRAS, 451, 2352

Kelly B. C., Merloni A., 2012, Advances in Astronomy, 2012, 970858

Khandai N., Di Matteo T., Croft R., Wilkins S., Feng Y., Tucker E., DeGraf C., Liu M.-S., 2015, MNRAS, 450, 1349

Kistler M. D., Yüksel H., Beacom J. F., Hopkins A. M., Wyithe J. S. B., 2009, ApJ, 705, L104

Labbé I., et al., 2013, ApJ, 777, L19

Latif M. A., Ferrara A., 2016, Publ. Astron. Soc. Australia, 33, e051

Latif M. A., Schleicher D. R. G., Schmidt W., Niemeyer J. C., 2013, MNRAS, 436, 2989

Latif M. A., Schleicher D. R. G., Hartwig T., 2016, MNRAS, 458, 233

Lodato G., Natarajan P., 2006, MNRAS, 371, 1813

Lodato G., Natarajan P., 2007, MNRAS, 377, L64
Lupi A., Colpi M., Devecchi B., Galanti G., Volonteri M., 2014, MNRAS, 442,3616

Lupi A., Volonteri M., Decarli R., Bovino S., Silk J., Bergeron J., 2019, MNRAS, 488, 4004

Lupi A., Haiman Z., Volonteri M., 2021, MNRAS, 503, 5046

Madau P., Dickinson M., 2014, ARA\&A, 52, 415

Madau P., Haardt F., Dotti M., 2014, ApJ, 784, L38

Mancini M., Schneider R., Graziani L., Valiante R., Dayal P., Maio U., Ciardi B., Hunt L. K., 2015, MNRAS, 451, L70

Mancini M., Schneider R., Graziani L., Valiante R., Dayal P., Maio U., Ciardi B., 2016, MNRAS, 462, 3130

Matsuoka Y., et al., 2018, ApJ, 869, 150

Matsuoka Y., et al., 2019, The Astrophysical Journal Letters, 872, L2

Mayer L., 2018, arXiv preprint arXiv:1807.06243

Mayer L., 2019, Super-Eddington accretion; flow regimes and conditions in high-z galaxies. pp 195-222, doi:10.1142/9789813227958_0011

Mayer L., Bonoli S., 2019, Reports on Progress in Physics, 82, 016901

Mayer L., Kazantzidis S., Madau P., Colpi M., Quinn T., Wadsley J., 2007, Science, 316, 1874

Mayer L., Kazantzidis S., Escala A., Callegari S., 2010, Nature, 466, 1082

Mayer L., Fiacconi D., Bonoli S., Quinn T., Roškar R., Shen S., Wadsley J., 2015, ApJ, 810, 51

Mazzucchelli C., et al., 2017, ApJ, 849, 91

McAlpine S., Bower R. G., Harrison C. M., Crain R. A., Schaller M., Schaye J., Theuns T., 2017, MNRAS, 468, 3395

McAlpine S., Bower R. G., Rosario D. J., Crain R. A., Schaye J., Theuns T., 2018, MNRAS, 481, 3118

McGreer I. D., Fan X., Jiang L., Cai Z., 2018, AJ, 155, 131

Merlin E., et al., 2019, MNRAS, 490, 3309

Merloni A., Heinz S., 2008, MNRAS, 388, 1011

Merloni A., et al., 2014, MNRAS, 437, 3550

Miyaji T., et al., 2015, ApJ, 804, 104

Mortlock D. J., et al., 2011, Nature, 474, 616

Natarajan P., Pacucci F., Ferrara A., Agarwal B., Ricarte A., Zackrisson E., Cappelluti N., 2017, ApJ, 838, 117

Niida M., et al., 2020, ApJ, 904, 89

Oesch P. A., et al., 2014, ApJ, 786, 108

Omukai K., 2001, ApJ, 546, 635

Omukai K., Tsuribe T., Schneider R., Ferrara A., 2005, The Astrophysical Journal, 626, 627

Omukai K., Schneider R., Haiman Z., 2008, The Astrophysical Journal, 686, 801

Onoue M., et al., 2019, ApJ, 880, 77

Pacucci F., Ferrara A., Volonteri M., Dubus G., 2015, MNRAS, 454, 3771

Pacucci F., Ferrara A., Grazian A., Fiore F., Giallongo E., Puccetti S., 2016, MNRAS, 459, 1432

Pacucci F., Natarajan P., Volonteri M., Cappelluti N., Urry C. M., 2017, arXiv preprint arXiv:1710.09375

Parkinson H., Cole S., Helly J., 2008, MNRAS, 383, 557

Parsa S., Dunlop J. S., McLure R. J., 2018, MNRAS, 474, 2904

Pezzulli E., Valiante R., Schneider R., 2016, MNRAS, 458, 3047

Pezzulli E., Valiante R., Orofino M. C., Schneider R., Gallerani S., Sbarrato T., 2017a, MNRAS, 466, 2131

Pezzulli E., Volonteri M., Schneider R., Valiante R., 2017b, Monthly Notices of the Royal Astronomical Society, 471, 589

Pfister H., Lupi A., Capelo P. R., Volonteri M., Bellovary J. M., Dotti M., 2017, MNRAS, 471, 3646

Pfister H., Volonteri M., Dubois Y., Dotti M., Colpi M., 2019, MNRAS, 486, 101

Piana O., Dayal P., Volonteri M., Choudhury T. R., 2021, MNRAS, 500, 2146

Planck Collaboration et al., 2018, arXiv e-prints, p. arXiv:1807.06209

Pons E., McMahon R. G., Simcoe R. A., Banerji M., Hewett P. C., Reed S. L., 2019, MNRAS, 484, 5142

Reed S. L., et al., 2017, MNRAS, 468, 4702

Reed S. L., et al., 2019, MNRAS, 487, 1874

Regan J. A., Haehnelt M. G., 2009, MNRAS, 396, 343 
Regan J. A., Downes T. P., Volonteri M., Beckmann R., Lupi A., Trebitsch M., Dubois Y., 2019, MNRAS, 486, 3892

Regan J. A., Haiman Z., Wise J. H., O'Shea B. W., Norman M. L., 2020 , arXiv e-prints, p. arXiv:2006.14625

Reines A. E., Volonteri M., 2015, ApJ, 813, 82

Reinoso B., Schleicher D. R. G., Fellhauer M., Klessen R. S., Boekholt T. C. N., 2018, A\&A, 614, A14

Reinoso B., Schleicher D., Fellhauer M., Klessen R., Boekholt T., Vergara M., Alister Seguel P., 2019, Boletin de la Asociacion Argentina de Astronomia La Plata Argentina, 61, 154

Ricarte A., Natarajan P., 2018a, MNRAS, 474, 1995

Ricarte A., Natarajan P., 2018b, MNRAS, 481, 3278

Sakurai Y., Yoshida N., Fujii M. S., Hirano S., 2017, Monthly Notices of the Royal Astronomical Society, 472, 1677

Salvaterra R., Haardt F., Volonteri M., Moretti A., 2012, A\&A, 545, L6

Sassano F., Schneider R., Valiante R., Inayoshi K., Chon S., Omukai K., Mayer L., Capelo P. R., 2021, arXiv e-prints, p. arXiv:2106.08330

Schaye J., et al., 2015, MNRAS, 446, 521

Schenker M. A., et al., 2013, ApJ, 768, 196

Schneider R., Ferrara A., Natarajan P., Omukai K., 2002, The Astrophysical Journal, 571, 30

Schneider R., Omukai K., Inoue A. K., Ferrara A., 2006, Monthly Notices of the Royal Astronomical Society, 369, 1437

Schneider R., Omukai K., Bianchi S., Valiante R., 2012, MNRAS, 419, 1566

Shakura N. I., Sunyaev R. A., 1973, A\&A, 500, 33

Shankar F., Weinberg D. H., Miralda-Escudé J., 2009, ApJ, 690, 20

Shankar F., Crocce M., Miralda-Escudé J., Fosalba P., Weinberg D. H., 2010, ApJ, 718, 231

Shankar F., et al., 2016, MNRAS, 460, 3119

Shankar F., et al., 2020, Nature Astronomy, 4, 282

Shao Y., et al., 2017, ApJ, 845, 138

Shen Y., Liu X., 2012, ApJ, 753, 125

Shen Y., et al., 2011, ApJS, 194, 45

Shen Y., et al., 2019, ApJ, 873, 35

Shen X., Hopkins P. F., Faucher-Giguère C.-A., Alexander D. M., Richards G. T., Ross N. P., Hickox R. C., 2020, MNRAS, 495, 3252

Sheth R. K., Mo H. J., Tormen G., 2001, MNRAS, 323, 1

Sijacki D., Springel V., Di Matteo T., Hernquist L., 2007, MNRAS, 380, 877

Sijacki D., Vogelsberger M., Genel S., Springel V., Torrey P., Snyder G. F., Nelson D., Hernquist L., 2015, MNRAS, 452, 575

Sądowski A., 2009, ApJS, 183, 171

Somerville R. S., Hopkins P. F., Cox T. J., Robertson B. E., Hernquist L., 2008, MNRAS, 391, 481

Song M., et al., 2016, ApJ, 825, 5

Souza Lima R., Mayer L., Capelo P. R., Bortolas E., Quinn T. R., 2020, arXiv e-prints, p. arXiv:2003.13789

Springel V., et al., 2005, Nature, 435, 629

Stacy A., Bromm V., Lee A. T., 2016, MNRAS, 462, 1307

Stark D. P., Schenker M. A., Ellis R., Robertson B., McLure R., Dunlop J., 2013, ApJ, 763, 129

Sugimura K., Matsumoto T., Hosokawa T., Hirano S., Omukai K., 2020, arXiv e-prints, p. arXiv:2002.00012

Suh H., Civano F., Trakhtenbrot B., Shankar F., Hasinger G., Sanders D. B., Allevato V., 2020, ApJ, 889, 32

Takeo E., Inayoshi K., Ohsuga K., Takahashi H. R., Mineshige S., 2018, MNRAS, 476, 673

Tamburello V., Capelo P. R., Mayer L., Bellovary J. M., Wadsley J. W., 2017, MNRAS, 464, 2952

Tamfal T., Capelo P. R., Kazantzidis S., Mayer L., Potter D., Stadel J., Widrow L. M., 2018, ApJ, 864, L19

Tanaka T., Haiman Z., 2009, ApJ, 696, 1798

Tenneti A., Di Matteo T., Croft R., Garcia T., Feng Y., 2018, MNRAS, 474, 597

Tenneti A., Wilkins S. M., Di Matteo T., Croft R. A. C., Feng Y., 2019, MNRAS, 483, 1388

Thomas N., Davé R., Anglés-Alcázar D., Jarvis M., 2019, MNRAS, 487, 5764
Tremmel M., Karcher M., Governato F., Volonteri M., Quinn T. R., Pontzen A., Anderson L., Bellovary J., 2017, MNRAS, 470, 1121

Ueda Y., Akiyama M., Hasinger G., Miyaji T., Watson M. G., 2014, ApJ, 786, 104

Valiante R., Schneider R., Salvadori S., Bianchi S., 2011, Monthly Notices of the Royal Astronomical Society, 416, 1916

Valiante R., Schneider R., Maiolino R., Salvadori S., Bianchi S., 2012, MNRAS, 427, L60

Valiante R., Schneider R., Salvadori S., Gallerani S., 2014, Monthly Notices of the Royal Astronomical Society, 444, 2442

Valiante R., Schneider R., Volonteri M., Omukai K., 2016, Monthly Notices of the Royal Astronomical Society, 457, 3356

Valiante R., Agarwal B., Habouzit M., Pezzulli E., 2017, Publ. Astron. Soc. Australia, 34, e031

Valiante R., Schneider R., Graziani L., Zappacosta L., 2018a, MNRAS, 474, 3825

Valiante R., Schneider R., Zappacosta L., Graziani L., Pezzulli E., Volonteri M., 2018b, MNRAS, 476, 407

Valiante R., et al., 2020, MNRAS, 500, 4095

Vito F., et al., 2018, MNRAS, 473, 2378

Vogelsberger M., et al., 2014, MNRAS, 444, 1518

Volonteri M., 2010, The Astronomy and Astrophysics Review, 18, 279

Volonteri M., Natarajan P., 2009, MNRAS, 400, 1911

Volonteri M., Lodato G., Natarajan P., 2008, MNRAS, 383, 1079

Volonteri M., Dubois Y., Pichon C., Devriendt J., 2016, MNRAS, 460, 2979

Wang F., et al., 2018, ApJ, 869, L9

Wang F., et al., 2020, ApJ, 896, 23

Wang F., et al., 2021, ApJ, 907, L1

Weinberger R., et al., 2017, MNRAS, 465, 3291

Weingartner J. C., Draine B. T., 2001, ApJ, 548, 296

Willott C. J., et al., 2010a, AJ, 139, 906

Willott C. J., et al., 2010b, AJ, 140, 546

Wise J. H., Turk M. J., Abel T., 2008, ApJ, 682, 745

Wise J. H., Regan J. A., O'Shea B. W., Norman M. L., Downes T. P., Xu H., 2019, Nature, 566, 85

Woods T. E., et al., 2019, Publ. Astron. Soc. Australia, 36, e027

Wu X.-B., et al., 2015, Nature, 518, 512

Yang J., et al., 2020, ApJ, 897, L14

Yue B., Ferrara A., Salvaterra R., Xu Y., Chen X., 2013, MNRAS, 433, 1556

Zhang H., Behroozi P., Volonteri M., Silk J., Fan X., Hopkins P. F., Yang J., Aird J., 2021, arXiv e-prints, p. arXiv:2105.10474

Zhu Q., Li Y., Li Y., Maji M., Yajima H., Schneider R., Hernquist L., 2020, arXiv e-prints, p. arXiv:2012.01458

de Bennassuti M., Schneider R., Valiante R., Salvadori S., 2014, MNRAS, 445, 3039

de Bennassuti M., Salvadori S., Schneider R., Valiante R., Omukai K., 2017, MNRAS, 465, 926

This paper has been typeset from a $\mathrm{T}_{\mathrm{E}} \mathrm{X} / \mathrm{LAT}_{\mathrm{E}} \mathrm{X}$ file prepared by the author. 